\title{
Mass and radius determinations for five transiting M-dwarf stars.
}

\author{
Jose M. Fernandez ${ }^{1,2}$, David W. Latham ${ }^{1}$, Guillermo Torres ${ }^{1}$, Mark E. Everett ${ }^{3}$, Georgi \\ Mandushev $^{4}$, David Charbonneau ${ }^{1}$, Francis T. O'Donovan ${ }^{5}$, Roi Alonso ${ }^{6}$, Gilbert A. \\ Esquerdo $^{1}$, Carl W. Hergenrother ${ }^{7}$, Robert P. Stefanik ${ }^{1}$
}

\begin{abstract}
We have derived masses and radii for both components in five short-period single-lined eclipsing binary stars discovered by the TrES wide-angle photometric survey for transiting planets. All these systems consist of a visible F-star primary and an unseen M-star secondary $\left(M_{\mathrm{A}} \geq 0.8 M_{\odot}, M_{\mathrm{B}} \leq 0.45 M_{\odot}\right)$. The spectroscopic orbital solution combined with a high precision transit light curve for each system gives sufficient information to calculate the density of the primary star and the surface gravity of the secondary. The masses of the primary stars were obtained using stellar evolution models, which requires accurate determinations of metallicities and effective temperatures. In our case, the uncertainty in the metallicity of the primary stars is the most important limiting factor in order to obtain accurate results for the masses and radii of the unseen M-dwarf secondaries. The solutions were compared with results obtained by calculating the radius of the primary stars under the assumption of rotational synchronization with the orbital period and alignment between their spin axis and the axis of the orbit, using the observed broadening of the spectral lines as an indicator of stellar rotation. Four systems show an acceptable match between the two sets of results when their metallicity is allowed to vary around solar values $(-0.5 \leq[\mathrm{Fe} / \mathrm{H}] \leq+0.5)$, but one system shows a clear mismatch between
\end{abstract}

\footnotetext{
${ }^{1}$ Harvard-Smithsonian Center for Astrophysics, 60 Garden Street, Cambridge, MA 02138, USA; jfernand@cfa.harvard.edu.

${ }^{2}$ Department of Astronomy, Pontificia Universidad Católica, Casilla 306, Santiago 22, Chile.

${ }^{3}$ Planetary Science Institute, 1700 East Fort Lowell Road, Suite 106, Tucson, AZ 85719, USA.

${ }^{4}$ Lowell Observatory, 1400 W. Mars Hill Rd., Flagstaff, AZ 86001, USA.

${ }^{5}$ NASA Postdoctoral Program Fellow, Goddard Space Flight Center, 8800 Greenbelt Rd Code 690.3, Greenbelt, MD 20771, USA.

${ }^{6}$ Laboratoire d'Astrophysique de Marseille (UMR 6110), Technople de Marseille-Etoile, F-13388 Marseille cedex 13, France.

${ }^{7}$ Lunar and Planetary Laboratory, University of Arizona, Tucson, AZ 85719, USA.
} 
the two solutions, which may indicate the absence of synchronization or a misalignment between the rotational and orbital axis. When compared to low-mass stellar evolution models, the derived masses and radii of the unseen $M$ dwarfs are inconsistent (three only marginally) with the predicted values, with all of the radii being larger than expected for their masses. These results confirm the discrepancy shown in previous work between the predicted and observed radii on low-mass binary stars. This work also shows that reliance on the assumption of synchronization to derive the mass and radius of stars in eclipsing single-lined $\mathrm{F}+\mathrm{M}$ binaries is a useful tool, but may not always be warranted and should be carefully tested against stellar evolution models.

Subject headings: binaries: eclipsing — binaries: spectroscopic — stars: fundamental parameters — stars: low-mass — stars: rotation — stars: ages

\section{Introduction}

Dynamical determination of fundamental properties of stars has played a key role in our understanding of stellar physics. Through careful observations of eclipsing double-lined spectroscopic binaries it is possible to obtain results with a precision as high as $1 \%$ on their masses and radii, or even better in some cases. These quantities provide strong constraints on stellar evolution models. Low-mass stars $\left(M \leq 0.5 M_{\odot}\right)$ are particularly difficult to study, mainly because of the low probability of finding eclipsing systems and also due to their very low intrinsic brightness. Precise constraints on their masses and radii are essential to model changes in their equation of state, which goes from being close to an ideal gas for the more massive stars, to a partially degenerate electron gas for M dwarfs (Baraffe et al. 1998; Chabrier et al. 2000). Thus, stars near the bottom of the main sequence pose a real challenge to stellar astronomers.

To date there are only eight $M$ dwarfs (in four binary systems) with published masses between 0.2 and $0.6 M_{\odot}$ that have their masses and radii measured to better than 3 percent: CM Draconis (Morales et al. 2008), YY Geminorum (Torres \& Ribas 2002), CU Cancri (Ribas 2003) and GU Bootis (López-Morales \& Ribas 2005). Remarkably, all these stars are consistently larger than predicted by low-mass stellar evolution models, and recent results point towards induced stellar activity as the cause of the larger radii observed in low-mass double-lined eclipsing binaries (López-Morales 2007; Chabrier et al. 2007).

Because the numbers are so small, every additional well studied low-mass star is important in our understanding of their fundamental properties. In recent years, the growing 
number of short-period single-lined eclipsing binaries (SEBs) with F-star primaries and Mdwarf secondaries (hereafter $\mathrm{F}+\mathrm{M}$ binaries) identified by photometric surveys for transiting planets promises to provide a way to fill the low-mass domain gap in the mass-radius diagram (Bouchy et al. 2005; Pont et al. 2005a, b, 2006; Beatty et al. 2007). Their large amplitude orbital radial velocities (several $\mathrm{km} \mathrm{s}^{-1}$ ) and transit depths between $1 \%$ and $4 \%$ make their follow up a reasonable task for 1-2-m class telescopes on stars brighter than $12^{\text {th }}$ mag. However, unlike double-lined eclipsing binaries, these systems do not directly provide masses and radii because the radial velocity curve can be only measured for the primary star (since only spectral lines from that star are visible). Only the mean stellar density for the primary and the surface gravity for the secondary star can be obtained directly from the observables.

One way to obtain a full solution for the masses and radii of a single-lined system is to use stellar evolution models to estimate the mass of the primary star based on its luminosity, effective temperature and metallicity. The same approach has been used to calculate the mass and radius of transiting extrasolar planets, which deliver uncertainties typically between $5 \%$ and $10 \%$ for the masses, depending on how well constrained the atmospheric properties of the primary stars are.

However, a different method can be used to solve for the masses and radii of SEBs, which is virtually independent of stellar evolution models. In $\mathrm{F}+\mathrm{M}$ binaries, the gravitational interaction between the primary and secondary star is at least two orders of magnitude stronger than in planetary systems. For binaries with short orbital periods $(\lesssim 6 \mathrm{~d})$ and measured eccentricities close to zero, one may in principle assume that two processes have already taken place due to tidal forces: synchronization between the orbital motion and stellar rotation, and alignment between the orbital and rotational axis (Zahn 1977; Hut 1981). The timescales for synchronization and axis alignment can be 50 or 100 times shorter than the time it takes to circularize the orbits, which can range from less than 100 Myr for very short period binaries $(1-2 \mathrm{~d})$ to more than 1 Gyr for binaries with longer periods (5-6 d). If synchronization and alignment have taken place, the radius of the primary star can be obtained by combining the rotational velocity derived from the observed broadening of the spectral lines with the orbital period and the inclination of the orbit, setting the scale of the system. This approach depends on the predictions from stellar models only in minor ways: limb darkening coefficients are needed for the detailed analysis of the eclipse light curve, and the rotational broadening that is derived from the observed spectra can depend weakly on the metallicity that is adopted. A precision (but not necessarily accuracy) of $5 \%$ or better in the mass and radius is possible if radial and rotational velocities are good to $2 \%$ and transit photometry is good to $1 \%$, as will be shown in this work.

Interestingly, it has been noted previously in the literature that the assumption of 
synchronization does not always seem to hold when tested in detail. Two short-period lowmass transiting M dwarfs discovered by OGLE (Pont et al. 2005b, 2006) delivered unrealistic properties for the primary stars if synchronization or pseudo synchronization was assumed. Their mass and radius had to be obtained from an estimation of the primary star properties using stellar isochrones, with final errors near $10 \%$ for the masses and $7 \%$ for the radii.

The goal of this paper is to contribute to our understanding of the fundamental properties of low mass stars. To accomplish this, we have determined the mass and radius for the components of five eclipsing single-lined F+M binaries identified by the TrES wide-angle transiting planet survey (TrES, Alonso et al. 2004). The next section describes the discovery and follow up observations of our targets. In section 3 we describe the models that were fitted to the data. In section 4 we present our results and discuss some implications of this study, in particular for the dynamical evolution of short period binary stars, and the usefulness of these systems to obtain a precise characterization of low-mass stars.

\section{Observations and Data Reduction}

\subsection{Detection through TrES Photometry}

Transit events for the systems studied in this paper where detected early on during regular operations of the Trans-Atlantic Exoplanet Survey network (TrES), an arrangement of three 10-cm telescopes distributed in longitude. The three network nodes are: the STARE telescope (Observatorio del Teide of the Instituto de Astrofísica de Canarias, Spain), the Sleuth Telescope (Palomar Observatory, California, USA), and the Planet Search Survey Telescope (Lowell Observatory, Arizona, USA). More than 30 fields of $5.7^{\circ} \times 5.7^{\circ}$ were monitored between the years 2003 and 2008. The data from each telescope were processed separately, as described by Dunham et al. (2004). The binned light curves were analyzed using the box-fitting transit search algorithm of Kovács et al. (2002) to find periodic signals consistent with the passage of a Jupiter-sized object across the disk of a solar-like star. Hundreds of objects were flagged as planetary candidates because of their shallow transit depth and the lack of a secondary eclipse or significant out-of-transit photometric variations. In general, these preliminary candidates were expected to fall within three categories: transiting planets, photometric false detections, and astrophysical false positives. Of all these candidates we selected five for the present work, based on their spectroscopic properties and the high-precision follow-up observations we were able to gather, as described below. Coordinates, visual magnitudes, and near-infrared colors for these five objects are given in Table 1. 


\section{2. $\quad$ Follow-up Spectroscopy}

A common strategy for following up transiting-planet candidates identified by widefield photometric surveys is to start with an initial spectroscopic reconnaissance, to see if there is evidence for a stellar companion that might be responsible for the observed light

curve. For this we used the CfA Digital Speedometers (Latham 1992) on the 1.5-m Wyeth Reflector at the Oak Ridge Observatory in the town of Harvard, Massachusetts, USA and on the 1.5-m Tillinghast Reflector at the Fred L. Whipple Observatory on Mount Hopkins, Arizona, USA. We obtained single-order echelle spectra in a wavelength window of $45 \AA$ centered at $5187 \AA$, with a resolution of $8.5 \mathrm{~km} \mathrm{~s}^{-1}$, and a typical signal-to-noise ratio per resolution element of 15 to 20. For slowly rotating solar-type stars these spectra deliver radial velocities accurate to about $0.5 \mathrm{~km} \mathrm{~s}^{-1}$, which is sufficient to detect orbital motion due to companions with masses down to about 5 or $10 M_{\mathrm{J}}$ for orbital periods of a few days. A detailed description of the spectroscopic data reduction can be found elsewhere (Alonso et al. 2004; Beatty et al. 2007; Latham et al. 2008). From the first set of spectra it is clear when candidates are not planets. If large variations in the radial velocities are observed, the explanation for the photometric signal is usually an eclipsing binary star (both signals should have a consistent periodicity and phasing). A total of 26 orbital solutions were derived for SEBs from the spectroscopic observations of planetary candidates. Orbital periods for these systems range from 1.2 days to 15.3 days, and eccentricities range from 0.0 to 0.5 (see Figure 1). The rotational broadening of the spectral lines, to be described in Section 2.4, also varies significantly reaching $V_{\text {rot }} \sin i_{\text {rot }}$ values of up to $60 \mathrm{~km} \mathrm{~s}^{-1}$ or more (see Figure 2). The phased radial velocities of the five SEBs studied in this work are shown in Figure 3, and the individual radial velocity measurements are presented in Table 2 to Table 6. Table 7 gives a comprehensive summary of the orbital solutions obtained from the spectroscopy.

\subsection{Follow-up KeplerCam Photometry}

In single-lined eclipsing systems is not possible to perform direct measurements of the radius of the secondary component. The only closely related observable quantity is the radius ratio between the objects, obtained through careful analysis of the photometric transit of the system. The main difficulty in obtaining a complete, high signal-to-noise transit light curve is the necessity of continuous excellent weather during several hours of observation. Because of this limitation, we scheduled times-series photometric observations only for those systems in which the assumption of orbit-rotation synchronization seemed to be secure (short orbital periods, and eccentricities close to zero), and with values of $V_{\text {rot }} \sin i_{\text {rot }}$ larger than 
about $10 \mathrm{~km} \mathrm{~s}^{-1}$, to avoid the accumulation of larger errors in the determination of the radius of the primary star as the spectroscopic resolution was approached. We used the predicted eclipse times from our spectroscopic orbits to schedule observations of the systems that passed this test, and successfully observed full transits of five SEBs. To provide a high-quality light curve for the analysis of the primary eclipse of each system, we used KeplerCam on the 1.2-m telescope at the Fred L. Whipple Observatory on Mount Hopkins, Arizona. KeplerCam utilizes a monolithic $4 \mathrm{~K} \times 4 \mathrm{~K}$ Fairchild $486 \mathrm{CCD}$ that gives a $23^{\prime} \times 23^{\prime}$ field and a pixel size of $0.68^{\prime \prime}$ when the binning is $2 \times 2$. To minimize limb darkening effects on the shape of the transit light curves, observations were made using long wavelength filters (Sloan $i$ and $z$ bands). Relative aperture photometry was performed to obtain the light curves. We iteratively selected comparison stars by removing any that showed unusual noise or variability. The typical RMS residual for the five light curves varied between 0.0010 and 0.0018 in relative flux units. Table 8 gives a summary of the photometric observations, with information about dates, pass bands, exposure times, cadence, air-mass, FWHM and RMS. Figure 4 shows the observed light curves in scaled relative flux, and samples of the observations are listed in Table 9. We intend for this table to appear in its entirety in the electronic version of the journal.

\subsection{Rotational Velocities, Effective Temperatures, and Metallicities}

The key to obtaining masses and radii under the assumption of synchronization is the determination of $V_{\text {rot }} \sin i_{\text {rot }}\left(i_{\text {rot }}\right.$ being the inclination angle between the rotational axis of the primary star and the line of sight), because when combined with the orbital period and orbital inclination, it sets the size of the primary star (assuming as well that $i_{\text {rot }}=i_{\text {orb }}$, the latter being the angle between the orbital axis and the line of sight). The projected

rotational velocity, along with the effective temperature and surface gravity of each star, were extracted from the same echelle spectra used to derive radial velocities. We cross-correlated our spectra against a library of synthetic spectra created by J. Morse using Kurucz model atmospheres (see, e.g., Latham et al. 2002) to estimate these properties for the primary stars for a given metallicity, which we fixed at four different values: $[\mathrm{Fe} / \mathrm{H}]=-1.0$, $-0.5,0.0$ and +0.5 . For each spectrum we looked for the metallicity-indexed model with the highest correlation, which gave a corresponding rotational velocity, temperature and surface gravity. The adopted values for correlation index $C$, projected rotational velocity $V_{\text {rot }} \sin i_{\text {rot }}$ and effective temperature $T_{\text {eff }}$ were obtained averaging over all the individual observations, after performing a three-sigma rejection. The internal error is the observed standard deviation divided by the square root of the number of measurements. In most cases, the average correlation index $\langle C\rangle$ is highest when solar metallicity is considered, but 
the typical error is generally larger than the differences. Thus this method cannot be used to obtain precise and accurate metallicities (see left panels of Figure 5). Because of the narrow wavelength coverage of our spectra, there is a clear dependence between the stellar properties (effective temperature in particular) and the adopted metallicity. To decrease the level of degeneracy, we iteratively used the surface gravity of the primary star obtained at the end of the procedure to be explained in Section 3.3.1 as an additional constraint for the cross correlation routine. Figure 5 shows the relation between metallicity, correlation index, effective temperature and rotational velocity for the five systems under study. Table 10 gives the values and uncertainties for the primary star atmospheric parameters for each adopted metallicity.

\section{Data Modeling}

\section{1. $\quad$ Light Curve Analysis}

Modeling of the light curves was carried out employing the formalism of Mandel \& Agol (2002), using a quadratic limb-darkening law,

$$
I_{\mu}=1-u_{1}(1-\mu)-u_{2}(1-\mu)^{2}
$$

where $I_{\mu}$ is the observed intensity relative to the center of the stellar disk and $\mu$ is the cosine of the angle between the line of sight and the normal to the stellar surface. The limb darkening coefficients $u_{1}$ and $u_{2}$ were taken from the tables of Claret (2004) adopting the metallicity-dependent values of $T_{\text {eff }}$ and $\log g$. The periods were held fixed and the orbits were assumed to be circular since the eccentricities obtained from the orbital solutions are close to zero for all systems. The fitted parameters were the radius ratio $R_{\mathrm{B}} / R_{\mathrm{A}}$, the reduced semi-major axis $a / R_{\mathrm{A}}$, and the impact parameter $b$, defined as $b=a / R_{\mathrm{A}} \cos i_{\text {orb }}$ where $i_{\text {orb }}$ is the inclination of the orbital plane to the line of sight. Here $R_{\mathrm{A}}$ and $R_{\mathrm{B}}$ correspond, respectively, to the radius of the primary and secondary star in each binary. The same notation is used for all other parameters in the text and equations that contain the $\mathrm{A}$ and B sub-indexes.

The best fit between the model and the data was found minimizing $\chi_{l c}^{2}$ :

$$
\chi_{l c}^{2}=\sum_{i=1}^{N_{f}}\left[\frac{f_{i}^{o b s}-f_{i}^{m o d}}{\sigma_{i}}\right]^{2}
$$

where $f_{i}^{o b s}$ and $f_{i}^{\text {mod }}$ are the observed and modeled relative fluxes observed at time $i$, and $\sigma_{i}$ 
is the error of each data point. The best values and uncertainties for the fitted parameters were obtained using a Markov Chain Monte Carlo simulation (MCMC). As described by Ford (2005) and Holman et al. (2006), in this method a random process is used to create a sequence of points in parameter space that approximates the studied probability distribution. This sequence or chain is generated by a jump function that adds a Gaussian random number to each parameter. The jump is executed if the new point has a $\chi_{l c}^{2}$ lower than the previous point. If $\chi_{l c}^{2}$ is larger, the jump is made with a probability equal to $\exp \left(-\Delta \chi_{l c}^{2}\right)$. If the jump is not made, the new point is a copy of the previous one. The relative sizes of the perturbations were set using the uncertainties obtained by direct inspection of $\chi_{l c}^{2}$ across the parameter space, as done in Beatty et al. (2007). The sizes of the jumps are set by requiring that $\sim 25 \%$ of the jumps are accepted. Four independent chains of 55000 points were created for each light-curve, starting from a point 5- $\sigma$ away from the optimal values obtained by direct inspection, and discarding the first $20 \%$ of the points to minimize initial condition effects. The four chains were combined to create one long sequence of points. The best-fit value and uncertainties for each parameter were obtained from the value interval centered on the median that contains $68 \%$ of the points ( $1-\sigma$ errors). The results derived using limbdarkening coefficients adopting solar metallicity are summarized in Table 11. The differences between these results and those adopting different metallicities are negligible compared to their errors.

\subsection{Density and Surface Gravity}

As shown previously by Seager \& Mallén-Ornelas (2003), Southworth et al. (2004) and Sozzetti et al. (2007), an approximation to the mean stellar density of the primary star $\left(\rho_{\mathrm{A}}\right)$ and the surface gravity of the secondary $\left(g_{\mathrm{B}}\right)$ can be derived directly from Newton's modified version of Kepler's third law and the mass function of the binary. The familiar expressions

$$
\begin{gathered}
a^{3}=\frac{G}{4 \pi^{2}}\left(M_{\mathrm{A}}+M_{\mathrm{B}}\right) P^{2} \\
M_{\mathrm{B}}=\left(\frac{P}{2 \pi G}\right)^{1 / 3} \frac{K_{\mathrm{A}}}{\sin i_{\text {orb }}}\left(M_{\mathrm{A}}+M_{\mathrm{B}}\right)^{2 / 3}
\end{gathered}
$$

can be combined and re-written in the following form:

$$
\rho_{\mathrm{A}}=\frac{3 \pi}{G P^{2}}\left(a / R_{\mathrm{A}}\right)^{3}-\rho_{\mathrm{B}}\left(R_{\mathrm{B}} / R_{\mathrm{A}}\right)^{3}
$$




$$
g_{\mathrm{B}}=\frac{2 \pi}{P} \frac{K_{\mathrm{A}}}{\sin i_{\mathrm{orb}}}\left(\frac{a / R_{\mathrm{A}}}{R_{\mathrm{B}} / R_{\mathrm{A}}}\right)^{2}
$$

where $\rho_{\mathrm{B}}$ is the density of the secondary star. The expression for the mass function in eq. 44 assumes zero eccentricity, which is a good approximation in our case. This assumption is

also supported by studies such as those of Lucy \& Sweeney (1971) and Lucy (2005), which show that for single-lined binaries, small observed eccentricities $(e<0.1)$ are generally not statistically significant.

Using the parameters obtained earlier from modeling the transit light curves $\left(R_{\mathrm{B}} / R_{\mathrm{A}}\right.$, $\left.a / R_{\mathrm{A}}, b\right)$, along with the measured orbital parameters $\left(P, K_{\mathrm{A}}\right)$, we may restrict the location of the stars in each system on the mass-radius diagram to unique curves of constant stellar density for the primary, and constant surface gravity for the secondary, which are described completely by the observables (see, e.g. Beatty et al. 2007):

$$
\begin{aligned}
& M_{\mathrm{A}}=\frac{4 \pi^{2}}{G P^{2}}\left(a / R_{\mathrm{A}}\right)^{3}\left(1-\frac{P K_{\mathrm{A}}}{2 \pi\left(1-\left(b^{2} /\left(a / R_{\mathrm{A}}\right)^{2}\right)\right)^{1 / 2}\left(a / R_{\mathrm{A}}\right) R_{\mathrm{A}}}\right) R_{\mathrm{A}}^{3} \\
& M_{\mathrm{B}}=\frac{2 \pi}{G P}\left(\frac{a / R_{\mathrm{A}}}{R_{\mathrm{B}} / R_{\mathrm{A}}}\right)^{2} \frac{K_{\mathrm{A}}}{\left(1-\left(b^{2} /\left(a / R_{\mathrm{A}}\right)^{2}\right)\right)^{1 / 2}} R_{\mathrm{B}}^{2}
\end{aligned}
$$

where $\sin i_{\text {orb }}$ has been re-written in terms of the observables $a / R_{\mathrm{A}}$ and $b$. These expressions are essentially model-independent and are very useful for the following reason: if an independent measurement of the mass or the radius of the primary star (or secondary star) is made, it is possible to calculate a full solution for the system (making use of the radius ratio $\left.R_{\mathrm{B}} / R_{\mathrm{A}}\right)$.

\subsection{Mass and radius determinations}

As mentioned in Section 2.4, there is a clear dependency between the adopted metallicity of the primary star and its atmospheric properties derived from our spectra, particularly the effective temperature. A metallicity close to solar gave the best match between the observed spectra and the models in most cases, but it is not possible to independently obtain an accurate abundance for these stars from our data alone. To address this problem, in the following we model the data for the five systems assuming four different metallicities: $[\mathrm{Fe} / \mathrm{H}]=-1.0,-0.5,0.0,+0.5$. 


\subsubsection{System solution from stellar isochrones}

In order to obtain the mass and radius for the unseen $\mathrm{M}$ dwarf, we estimated the mass of the primary star using the Yonsei-Yale stellar evolution models (Yi et al. 2001; Demarque et al. 2004), following the procedure of Torres et al. (2008). For this purpose we relied on the adopted metallicity and the temperature obtained from the spectra. Parallaxes have not been measured for these targets, so instead of the luminosities of the stars, we used the parameter $a / R_{\mathrm{A}}$ derived from the modeling of the light curves, which is closely related to the mean stellar density.

The observed quantity $a / R_{\mathrm{A}}$ can be compared directly to the models re-writing eq. 3 ;

$$
a / R_{\mathrm{A}}=\left(\frac{G}{4 \pi^{2}}\right)^{1 / 3} \frac{P^{2 / 3}}{R_{\mathrm{A}}}\left(M_{\mathrm{A}}+M_{\mathrm{B}}\right)^{1 / 3}
$$

The mass of the secondary star is not know a priori, but an approximate value sufficient for the present purpose can be estimated using eq. 8 and the isochrones from Baraffe et al. (1998) given that the age dependency is weak for low-mass stars. Once the secondary star mass has been derived, the process can be repeated until convergence.

The Yonsei-Yale isochrones were interpolated to a fine grid in metallicity and age and compared point by point with the measured values of $T_{\text {eff }}$ and $a / R_{\mathrm{A}}$. As noted previously, the range of metallicities explored was $[\mathrm{Fe} / \mathrm{H}]=-1.0,-0.5,0.0,+0.5$. We adopted an arbitrary error of $\sigma_{[\mathrm{Fe} / \mathrm{H}]}= \pm 0.2$, meant to illustrate the behavior of the results over a wide range of metallicities in a compact way. The internal error for $T_{\text {eff }}$ was increased to account for the dependency with $[\mathrm{Fe} / \mathrm{H}]$ in the mentioned range, from the nominal precision at a given metallicity of about $50 \mathrm{~K}$ to a more conservative $200 \mathrm{~K}$.

Each point on the isochrones that was consistent with $[\mathrm{Fe} / \mathrm{H}], T_{\text {eff }}$ and $a / R_{\mathrm{A}}$ within their errors was recorded and a likelihood given by $L=\exp \left(-\chi_{i s o}^{2} / 2\right)$ was calculated for it, where

$$
\chi_{i s o}^{2}=\left(\frac{\Delta[\mathrm{Fe} / \mathrm{H}]}{\sigma_{[\mathrm{Fe} / \mathrm{H}]}}\right)^{2}+\left(\frac{\Delta T_{\mathrm{eff}}}{\sigma_{T_{\mathrm{eff}}}}\right)^{2}+\left(\frac{\Delta\left(a / R_{\mathrm{A}}\right)}{\sigma_{a / R_{\mathrm{A}}}}\right)^{2} .
$$

The $\Delta$ symbols represent the difference between the observed and model values for each quantity. The possible values for $L$ range between 1 (an exact match between observations and models) and 0.22 (the worst acceptable match). The best fit value for each stellar parameter was obtained by adding all matches, weighted by their corresponding normalized likelihood $L$. The adopted errors for the fitted parameters (mass and age) come from their 
range among the accepted points on the isochrones. In the case of solar metallicity, primary masses range from $1.3 M_{\odot}$ to $1.6 M_{\odot}$, with uncertainties ranging from $8 \%$ to $13 \%$. Ages range from 0.7 Gyr to 4.0 Gyr, with large uncertainties, from $25 \%$ to $100 \%$ in some cases. For lower metallicities, more massive and older stars are obtained. The opposite effect is observed for higher abundances.

With $M_{\mathrm{A}}$ known, we then obtained $M_{\mathrm{B}}$ and $a$ by iteration of equations 3 and 4 . The value of $a$ in combination with $a / R_{\mathrm{A}}$ and $R_{\mathrm{B}} / R_{\mathrm{A}}$ allowed us to obtain consistent values for $R_{\mathrm{A}}$ and $R_{\mathrm{B}}$. At this point, we used the newly derived surface gravity of the primary star $\log g_{\mathrm{A}}$ as an additional constraint in the determination of $\langle C\rangle, T_{\text {eff }}$ and $V_{\text {rot }} \sin i_{\text {rot }}$ as explained in Section 2.4. We iterated this procedure until convergence.

To estimate the errors for $R_{\mathrm{A}}, R_{\mathrm{B}}$, and $M_{\mathrm{B}}$, we used the MCMC chains generated in the course of modeling the transiting light curves. For each element of the chain a solution was calculated using the corresponding values of $R_{\mathrm{B}} / R_{\mathrm{A}}, a / R_{\mathrm{A}}$ and $b$ together with random values for $P$ and $K_{\mathrm{A}}$, normally distributed around the observed values with $\sigma$ equal to the measured uncertainties. In this way we obtained a probability distribution for the masses

and radii, from which we extracted the median and $68 \%$ confidence limits $(1-\sigma)$ and adopted them as best values and errors, respectively.

For solar metallicity, secondary masses range from $0.28 M_{\odot}$ to $0.43 M_{\odot}$, and secondary radii range from $0.28 R_{\odot}$ to $0.40 R_{\odot}$. The uncertainties in $M_{\mathrm{B}}$ range from $5 \%$ to $8 \%$, and those in $R_{\mathrm{B}}$ from $3 \%$ to $5 \%$. For sub-solar composition the masses and radii are smaller, and the opposite effect is observed for higher abundances.

The resulting masses and radii from the isochrone modeling of the primary stars are shown in Figure 6 with filled circles. For some cases of very high or low metallicity, no consistent solution was found based on stellar models, which explains some missing results in the figures. In these cases, the value for $\log g$ from the nearest consistent solution was used to better constrain the atmospheric properties of the primary star (Section 2.4).

\subsubsection{System solution from orbit-rotation synchronization}

The uncertainty in the determination of metallicities for the primary stars has significant consequences when estimating their masses using stellar models. To overcome this serious limitation, we computed masses and radii based on the assumption of synchronization and co-alignment between the primary star's rotational axis and the axis of the orbit.

From the rotational velocity, orbital period, and orbital inclination, it is possible to 
calculate the radius of the primary star $R_{\mathrm{A}}$ using the expression

$$
R_{\mathrm{A}}=\frac{P}{2 \pi\left(1-\left(b^{2} /\left(a / R_{\mathrm{A}}\right)\right)^{2}\right)^{1 / 2}}\left(V_{\mathrm{rot}} \sin i_{\mathrm{rot}}\right)
$$

which is the generalization of the geometric relation $P_{\text {rot }}=\frac{2 \pi R}{V_{\text {rot }}}$ for an impact parameter $b$ other than zero, under the assumption of synchronization $\left(P=P_{\text {orb }}=P_{\text {rot }}\right)$ and alignment between the rotation and orbital axes $\left(i_{\text {orb }}=i_{\text {rot }}\right)$. The radius ratio $\left(R_{\mathrm{B}} / R_{\mathrm{A}}\right)$ is then used to infer the size of secondary star. Once both radii are known, they can be incorporated into equations 7 and 8 to obtain full expressions for the masses.

For the error propagation we adopted uncertainties in $V_{\text {rot }} \sin i_{\text {rot }}$ as described in Sect. 2.4. We verified that for each of our five systems this error was larger than the variations that come from the correlation between $V_{\text {rot }} \sin i_{\text {rot }}$ and $[\mathrm{Fe} / \mathrm{H}]$ over the range of metallicities considered here. The final values and errors for the masses and radii of both components were obtained using the MCMC distributions from the light curve fits, incorporating the errors of $a / R_{\mathrm{A}}, R_{\mathrm{B}} / R_{\mathrm{A}}, b, P, V_{\text {rot }} \sin i_{\text {rot }}$ and $K_{\mathrm{A}}$ in a way analogous to the procedure used for the parameters inferred by using stellar isochrones.

For the primary stars, masses range from $0.8 M_{\odot}$ to $1.5 M_{\odot}$ (with errors between $5 \%$ and $15 \%$ ) and radii range from $1.1 R_{\odot}$ to $1.8 R_{\odot}$ (errors of $2-5 \%$ ). Among the secondary stars, masses range from $0.2 M_{\odot}$ to $0.35 M_{\odot}$ (with errors between $3 \%$ and $10 \%$ ) and radii range from $0.25 R_{\odot}$ to $0.35 R_{\odot}$ (errors of $2-5 \%$ ). The masses and radii for the primary stars obtained from the assumption of synchronization are shown in Figures 6 with filled triangles.

\section{Results and Discussion}

We begin by comparing the results obtained for the primary stars using stellar isochrones with those from synchronization assumptions. Figure 6 shows masses and radii vs. metallicity for every system. We remind the reader that the error bars in the results derived from stellar models include the contribution from a conservative error in the adopted metallicity of each star.

For every system but one (T-Aur0-13378) there is a metallicity range where both sets of results are consistent, but outside this range the solutions diverge considerably. The results derived from estimating the mass of the primary star using models show a strong dependence on the adopted metallicity, not only because of its direct impact in the isochrones, but also because the effective temperature is highly correlated with it. The results derived from 
orbit-rotation synchronization have a small dependence on the adopted metallicity due to the weak correlation between the observed rotational velocity of the primary star and the adopted abundance.

Tidal theory predicts these kinds of systems (short period, near circular orbit) should be synchronized and their orbital and rotational axes co-aligned. Following the formalism of Hut (1981), we calculated the ratio of the orbital and rotational angular momentum $\alpha=\frac{q}{(1+q)} \frac{1}{r_{g}^{2}}\left(a / R_{\mathrm{A}}\right)^{2}$, where $q=M_{\mathrm{B}} / M_{\mathrm{A}}$ and $r_{g}$ is the gyration radius of the primary star $\left(r_{g}^{2}=\frac{I}{M_{\mathrm{A}} R_{\mathrm{A}}^{2}}\right.$, with $I$ being the moment of inertia). For our binaries, $\alpha$ is always larger than 70 , which means that the timescales for synchronization and alignment of the orbital and rotational axes are expected to be much shorter than the timescale for circularization. In order to have comparable timescales for the three processes, $\alpha$ must be between 5 and 10 (Hut 1981). A similar relation between timescales was obtained by Zahn (1977), and again synchronization is predicted to occur much more rapidly than circularization for these binary systems.

Even when evidence in favor of synchronization is strong, its reality is not always guaranteed. The F+M binary OGLE-TR-123, studied by Pont et al. (2006) has a very short period $(1.8 \mathrm{~d})$ and a circular orbit, but the solution obtained if synchronization is assumed does not match the properties of the primary star derived spectroscopically. In this case, the solution derived from synchronization implies a primary star which is not massive enough $\left(M_{\mathrm{A}}<0.9 M_{\odot}\right)$ to explain its observed high effective temperature $\left(T_{\text {eff }} \sim 6700 K\right)$.

The same inconsistency affects the system T-Aur0-13378, with a clear disagreement between the solutions based on the assumption of synchronization and those derived from stellar models. The main problem with the solution based on synchronization is the unrealistically low mass and radius inferred for the primary star, given the relatively high temperatures obtained from spectroscopy. In other words, the measured rotational velocity is too low, resulting in a small radius. There are various possible explanations for this behavior. The primary star in this system has the lowest density, lowest surface gravity and the highest mass for a given metallicity (when using models), if compared to the other four (see Table 11 and Table 12). If solar or lower-than-solar metallicity is adopted for the modeling, the resulting primary is an evolved F-star with $M_{\mathrm{A}}<1.6 M_{\odot}$ and $R_{\mathrm{A}}>2.0 M_{\odot}$. If this is the case, it could be that conservation of angular momentum during the expansion of the star has slowed down the surface rotation, and tidal forces have not been able to keep up. Also, the presence of a radiative envelope in the primary could be responsible for making tidal forces less efficient. The discrepancy between the two solutions could also be explained by a misalignment between the spin axis of the primary and the axis of the orbit, which is, however, not expected from the same theory that tells us that synchronization should be taking place. 
By taking advantage of the Rossiter-McLaughlin effect (Rossiter 1924; McLaughlin 1924) it should be possible to measure the projection of this angle on the plane of the sky (see, e.g., Winn et al. 2006), but one would still need the orthogonal projection along the line of sight to solve the orientation of the system completely. One way to independently determine the rotational period of the primary star would be to obtain high-quality light curves and measure the photometric variation outside of eclipse that might be produced by rotation and star spots. This would serve as a check on the assumption of tidal synchronization in the system. Unfortunately, $\mathrm{F}$ stars are likely to be too hot for spot activity to be detectable in their light curves by ground-based facilities (see, e.g., Hall 1994). Dedicated spaced-based surveys like CoRot (Baglin et al. 2006) and Kepler (Borucki et al. 2003; Basri et al. 2005) may be able to detect this signal in similar systems.

Inconsistent solutions between tidal theory and stellar evolution similar to the case of T-Aur0-13378 would be obtained as well for the systems T-Lyr1-01662, T-Lyr0-08070, and TCyg1-01385 if accurate spectroscopic determinations revealed the primary star metallicities to be greater than solar. The system T-Boo0-00080 behaves differently from the others, in the sense that high metallicities are necessary to match both set of results. If the primary star in this system has a metallicity lower than solar, the measured rotational velocity would be too high for the system to be synchronized, and could be evidence of an ongoing tidal process where the primary star is slowing down from a faster initial rotation.

In view of the importance of knowing accurately the metallicity of the primary stars, we have chosen to tabulate the full range of results obtained from their modeling using stellar isochrones corresponding to four different abundances (Table 12). Additionally, we have tabulated the results derived from the assumption of orbit-rotation synchronization that show the best match with those derived from stellar models (Table 13). Strictly speaking, the errors for the masses and radii obtained from modeling the primary star with stellar isochrones are partially arbitrary because of the adopted error on the metallicities. The errors for the results based on synchronization are realistic and are dominated by the uncertainty in the measured rotational velocity.

In Figure 7 we display the mass and radius of the M-dwarf secondaries of the systems T-Boo0-00080, T-Lyr1-01662, T-Lyr0-08070, and T-Cyg1-01385 using the synchronizationbased results from Table 13. The system T-Aur-013378 is shown using the model-based results $([\mathrm{Fe} / \mathrm{H}]=0.0 \pm 0.2)$ because its uncertain tidal configuration does not allow a reliable determination of the mass and radius of the secondary star when assuming orbit-rotation synchronization. We also show the results for the M dwarfs HAT-TR-205-013 (Beatty et al. 2007), OGLE-TR-106 (Pont et al. 2005a), OGLE-TR-122 (Pont et al. 2005b), and OGLETR-123 (Pont et al. 2006), which have the lowest measured mass and radius available in 
the current literature for main sequence stars. These four stars are also members of singlelined spectroscopic binary systems. As mentioned in the introduction and earlier in this section, for OGLE-TR-122 and OGLE-TR-123 the authors had to use stellar models to estimate the masses and radii of the primary rather than the assumption of synchronization or pseudo-synchronization (OGLE-TR-122 has an eccentric orbit), as the latter would have implied masses and radii inconsistent with the spectroscopic observations. We have included in Figure 7 eight published $M$ dwarfs in double-lined binary systems (see Section 1), for a direct comparison with our results.

From Figure 7 it appears that the values for the mass and radius of T-Boo0-00080-B and T-Lyr1-01662-B are formally inconsistent with theoretical predictions from the Lyon group (Baraffe et al. 1998). A marginal inconsistency is also observed for T-Lyr0-08070-B, T-Cyg101385-B and T-Aur-013378-B. Our M dwarfs have radii that are larger than predicted for the measured masses when adopting consistent metallicities and ages. For the system TBoo0-00080, with a best fit metallicity of $[\mathrm{Fe} / \mathrm{H}]=+0.5$, a proper comparison is not possible because of the lack of published isochrones for this composition. However, the solution (based on synchronization) for solar metallicity is practically the same as the one adopted, and in that case there is a clear inconsistency. This radius discrepancy has been documented now for a number of systems and in particular for several well-measured low-mass double-lined eclipsing binaries (also included in Figure 7), and is believed to reflect the fact that these systems do not evolve as isolated stars. The rapid rotation, caused by tidal synchronization, may lead to enhanced magnetic activity, which can manifest itself in two ways: a decrease in the efficiency of energy transport in these mostly convective stars, resulting in inflated stellar radii and cooler temperatures, and significant spot coverage, with similar consequences (see, e.g., López-Morales \& Ribas 2005; Ribas 2006; López-Morales 2007; Chabrier et al. 2007). In order to check for possible activity, we looked for corresponding X-ray sources in the ROSAT mission catalog (Voges et al. 1999), but no match was found for any of our targets.

The next step in this work is to collect enough photometric data to perform a similar analysis of the remaining 21 SEBs mentioned earlier. Most of th observed eccentricities are close to zero (Figure 1) and the distribution of rotational velocities vs. orbital period suggest that orbit-rotation synchronization should be taking place for many of these systems (see Figure 2). It would strengthen the results to have detailed abundances for all these targets in order to break the metallicity-temperature degeneracy that affects our analysis in its present form. The recently commissioned TRES instrument at FLWO (Szentgyorgyi \& Furész 2007; Devor et al. 2008) could provide suitable spectra for this purpose. Absolute luminosities derived from accurate parallaxes would significantly improve the determination of the mass and radius of the primary stars during the isochrone modeling. The best hope for good parallaxes is the GAIA mission (Battrick 1994, and references thereafter), to be launched in 
2011. With the extra observations mentioned above, it should be possible to unambiguously determine which SEBs are synchronized and which ones are not, providing a valuable set of results for testing tidal theory.

\section{Summary}

We have determined masses and radii for the components of five eclipsing single-lined binaries consisting of an $\mathrm{F}$ star and an unseen $\mathrm{M}$ dwarf, identified photometrically by the TrES wide-angle transiting planet survey. Our results are based on accurate spectroscopic orbital solutions and high precision light curves, and were obtained in two different ways: by modeling the primary star using stellar isochrones, and by estimating the size of the primary star using the measured value of $V_{\text {rot }} \sin i_{\text {rot }}$ together with the assumption of synchronization and alignment between the spin axis of the primary star and the orbital axis.

The near zero eccentricity of the orbits of these systems makes the assumption of synchronization reasonable, following the predictions from tidal theory. The consistency of the two sets of results depends strongly on the adopted atmospheric parameters of the primary stars during the modeling of their masses, in particular the metallicity. Even when four of the five systems show an acceptable match between the two sets of results, a definitive value for their masses and radii still depends on the accurate determination of their abundances. If we adopt the synchronization-based solutions that best match those obtained from stellar evolution models, we find that in four of the studied systems the results are inconsistent with low-mass stellar evolution models, with $\mathrm{M}$ dwarfs that are larger than predicted (two only marginally). This behavior has been documented previously for a number of $\mathrm{M}$ dwarfs in binary systems.

Our results, combined with indications from previous work, show that reliance on the assumption of synchronization to derive the mass and radius of stars in eclipsing single-lined $\mathrm{F}+\mathrm{M}$ is a useful tool, but may not always be warranted and should be carefully tested against stellar evolution models.

We thank Joe Zajac, Perry Berlind, and Mike Calkins for obtaining some of the spectroscopic observations; Bob Davis for maintaining the database for the CfA Digital Speedometers; and John Geary, Andy Szentgyorgyi, Emilio Falco, Ted Groner, and Wayne Peters for their contribution to making KeplerCam such an effective instrument for obtaining highquality light curves. We are also grateful to the anonymous referee for very helpful comments and suggestions. This research was supported in part by the Kepler Mission under NASA Cooperative Agreement NCC2-1390. GT acknowledges partial support from NSF grant 
AST-0708229.

\section{REFERENCES}

Alonso, R., Brown, T. M., Torres, G., Latham, D. W., Sozzetti, A., Mandushev, G., Belmonte, J. A., Charbonneau, D., Deeg, H. J., Dunham, E. W., O'Donovan, F. T., \& Stefanik, R. P. 2004, ApJ, 613, L153

Baglin, A., Auvergne, M., Boisnard, L., Lam-Trong, T., Barge, P., Catala, C., Deleuil, M., Michel, E., \& Weiss, W. 2006, in COSPAR, Plenary Meeting, Vol. 36, 36th COSPAR Scientific Assembly, 3749-+

Baraffe, I., Chabrier, G., Allard, F., \& Hauschildt, P. H. 1998, A\&A, 337, 403

Basri, G., Borucki, W. J., \& Koch, D. 2005, New Astronomy Review, 49, 478

Battrick, B. 1994, Horizon 2000 Plus. European Space Science in the 21st Century, ed. B. Battrick

Beatty, T. G., Fernández, J. M., Latham, D. W., Bakos, G. Á., Kovács, G., Noyes, R. W., Stefanik, R. P., Torres, G., Everett, M. E., \& Hergenrother, C. W. 2007, ApJ, 663, 573

Borucki, W. J., Koch, D. G., Basri, G. B., Caldwell, D. A., Caldwell, J. F., Cochran, W. D., Devore, E., Dunham, E. W., Geary, J. C., Gilliland, R. L., Gould, A., Jenkins, J. M., Kondo, Y., Latham, D. W., \& Lissauer, J. J. 2003, in Astronomical Society of the Pacific Conference Series, Vol. 294, Scientific Frontiers in Research on Extrasolar Planets, ed. D. Deming \& S. Seager, 427-440

Bouchy, F., Pont, F., Melo, C., Santos, N. C., Mayor, M., Queloz, D., \& Udry, S. 2005, A\&A, 431, 1105

Chabrier, G., Baraffe, I., Allard, F., \& Hauschildt, P. 2000, ApJ, 542, 464

Chabrier, G., Gallardo, J., \& Baraffe, I. 2007, A\&A, 472, L17

Claret, A. 2004, A\&A, 428, 1001

Demarque, P., Woo, J.-H., Kim, Y.-C., \& Yi, S. K. 2004, ApJS, 155, 667

Devor, J., Charbonneau, D., Torres, G., Blake, C. H., White, R. J., Rabus, M., O'Donovan, F. T., Mandushev, G., Bakos, G. Á., Fưrész, G., \& Szentgyorgyi, A. 2008, ApJ, 687, 1253 
Dunham, E. W., Mandushev, G. I., Taylor, B. W., \& Oetiker, B. 2004, PASP, 116, 1072

Ford, E. B. 2005, AJ, 129, 1706

Hall, D. S. 1994, Memorie della Societa Astronomica Italiana, 65, 73

Holman, M. J., Winn, J. N., Latham, D. W., O’Donovan, F. T., Charbonneau, D., Bakos, G. A., Esquerdo, G. A., Hergenrother, C., Everett, M. E., \& Pál, A. 2006, ApJ, 652, 1715

Hut, P. 1981, A\&A, 99, 126

Kovács, G., Zucker, S., \& Mazeh, T. 2002, A\&A, 391, 369

Latham, D. W. 1992, in Astronomical Society of the Pacific Conference Series, Vol. 32, IAU Colloq. 135: Complementary Approaches to Double and Multiple Star Research, ed. H. A. McAlister \& W. I. Hartkopf, 110-+

Latham, D. W., Bakos, G. Á., Torres, G., Stefanik, R. P., Noyes, R. W., Kovács, G., Pál, A., Marcy, G. W., Fischer, D. A., Butler, R. P., Sipocz, B., Sasselov, D. D., Esquerdo, G. A., Vogt, S. S., Hartman, J. D., Kovács, G., Lázár, J., Papp, I., \& Sári, P. 2008, ArXiv e-prints

Latham, D. W., Stefanik, R. P., Torres, G., Davis, R. J., Mazeh, T., Carney, B. W., Laird, J. B., \& Morse, J. A. 2002, AJ, 124, 1144

López-Morales, M. 2007, ApJ, 660, 732

López-Morales, M., \& Ribas, I. 2005, ApJ, 631, 1120

Lucy, L. B. 2005, A\&A, 439, 663

Lucy, L. B., \& Sweeney, M. A. 1971, AJ, 76, 544

Mandel, K., \& Agol, E. 2002, ApJ, 580, L171

McLaughlin, D. B. 1924, ApJ, 60, 22

Morales, J. C., Ribas, I., Jordi, C., Torres, G., Gallardo, J., Guinan, E. F., Charbonneau, D., Wolf, M., Latham, D. W., Anglada-Escudé, G., Bradstreet, D. H., Everett, M. E., O’Donovan, F. T., Mandushev, G., \& Mathieu, R. D. 2008, ArXiv e-prints

Pont, F., Bouchy, F., Melo, C., Santos, N. C., Mayor, M., Queloz, D., \& Udry, S. 2005a, A\&A, 438, 1123 
Pont, F., Melo, C. H. F., Bouchy, F., Udry, S., Queloz, D., Mayor, M., \& Santos, N. C. 2005b, A\&A, 433, L21

Pont, F., Moutou, C., Bouchy, F., Behrend, R., Mayor, M., Udry, S., Queloz, D., Santos, N., \& Melo, C. 2006, A\&A, 447, 1035

Ribas, I. 2003, A\&A, 398, 239

-. 2006, Ap\&SS, 304, 89

Rossiter, R. A. 1924, ApJ, 60, 15

Seager, S., \& Mallén-Ornelas, G. 2003, ApJ, 585, 1038

Southworth, J., Zucker, S., Maxted, P. F. L., \& Smalley, B. 2004, MNRAS, 355, 986

Sozzetti, A., Torres, G., Charbonneau, D., Latham, D. W., Holman, M. J., Winn, J. N., Laird, J. B., \& O’Donovan, F. T. 2007, ApJ, 664, 1190

Szentgyorgyi, A. H., \& Furész, G. 2007, in Revista Mexicana de Astronomia y Astrofisica Conference Series, Vol. 28, Revista Mexicana de Astronomia y Astrofisica Conference Series, ed. S. Kurtz, 129-133

Torres, G., \& Ribas, I. 2002, ApJ, 567, 1140

Torres, G., Winn, J. N., \& Holman, M. J. 2008, ApJ, 677, 1324

Voges, W., Aschenbach, B., Boller, T., Bräuninger, H., Briel, U., Burkert, W., Dennerl, K., Englhauser, J., Gruber, R., Haberl, F., Hartner, G., Hasinger, G., Kürster, M., Pfeffermann, E., Pietsch, W., Predehl, P., Rosso, C., Schmitt, J. H. M. M., Trümper, J., \& Zimmermann, H. U. 1999, A\&A, 349, 389

Winn, J. N., Johnson, J. A., Marcy, G. W., Butler, R. P., Vogt, S. S., Henry, G. W., Roussanova, A., Holman, M. J., Enya, K., Narita, N., Suto, Y., \& Turner, E. L. 2006, ApJ, 653, L69

Yi, S., Demarque, P., Kim, Y.-C., Lee, Y.-W., Ree, C. H., Lejeune, T., \& Barnes, S. 2001, ApJS, 136, 417

Zahn, J.-P. 1977, A\&A, 57, 383 
Table 1. Target Coordinates and Magnitudes

\begin{tabular}{llllll}
\hline \hline & T-Aur0-13378 & T-Boo0-00080 & T-Lyr1-01662 & T-Lyr0-08070 & T-Cyg1-01385 \\
\hline RA (2000) & $05: 05: 06.9$ & $14: 35: 54.5$ & $18: 59: 02.8$ & $19: 19: 03.7$ & $20: 15: 21.9$ \\
DEC (2000) & $+41: 26: 03$ & $+46: 35: 36$ & $+48: 36: 35$ & $+38: 40: 57$ & $+48: 17: 14$ \\
$V$ (app. mag) & 13.0 & 10.3 & 11.3 & 12.3 & 10.7 \\
$J-K_{\text {MMASS }}(\mathrm{mag})$ & 0.34 & 0.26 & 0.20 & 0.25 & 0.32 \\
\hline
\end{tabular}

Table 2. Individual Radial Velocities for T-Aur0-13378

\begin{tabular}{crr}
\hline \hline $\begin{array}{c}\text { HJD } \\
\text { (days })\end{array}$ & $\begin{array}{c}V_{\mathrm{rad}} \\
\left(\mathrm{km} \mathrm{s}^{-1}\right)\end{array}$ & $\begin{array}{c}\sigma\left(V_{\mathrm{rad}}\right) \\
\left(\mathrm{km} \mathrm{s}^{-1}\right)\end{array}$ \\
\hline 2453334.8407 & -2.91 & 1.59 \\
2453401.7953 & 16.80 & 2.25 \\
2453452.6211 & -18.19 & 2.00 \\
2453626.9868 & 24.28 & 2.04 \\
2453663.9614 & 9.94 & 1.57 \\
2453667.0023 & 36.17 & 1.37 \\
2453667.9707 & -12.89 & 2.10 \\
2453684.9318 & 29.61 & 1.66 \\
2453687.9154 & 43.39 & 1.65 \\
2453721.9235 & -10.47 & 1.66 \\
2453990.0023 & 1.47 & 1.66 \\
2454041.8878 & 40.07 & 1.90 \\
2454101.7741 & 32.28 & 3.05 \\
\hline
\end{tabular}


Table 3. Individual Radial Velocities for T-Boo0-00080

\begin{tabular}{crr}
\hline $\begin{array}{c}\text { HJD } \\
(\text { days })\end{array}$ & $\begin{array}{c}V_{\text {rad }} \\
\left(\mathrm{km} \mathrm{s}^{-1}\right)\end{array}$ & $\begin{array}{c}\sigma\left(V_{\text {rad }}\right) \\
\left(\mathrm{km} \mathrm{s}^{-1}\right)\end{array}$ \\
\hline 2453078.7253 & -41.87 & 1.11 \\
2453080.6658 & 1.13 & 0.78 \\
2453080.9194 & -16.58 & 0.88 \\
2453084.6469 & -15.00 & 1.16 \\
2453084.9011 & 3.86 & 1.09 \\
2453086.6660 & -43.42 & 1.30 \\
2453086.8937 & -35.05 & 1.90 \\
2453087.6528 & 16.50 & 1.05 \\
2453088.8989 & -41.79 & 1.58 \\
2453094.6495 & -26.89 & 1.90 \\
2453154.7784 & -34.38 & 1.27 \\
2453176.6766 & 20.67 & 1.51 \\
2453187.7755 & -32.58 & 1.16 \\
2453227.5743 & 19.77 & 1.09 \\
2453780.0387 & -45.40 & 1.62 \\
2453807.9210 & -45.37 & 1.54 \\
2453808.8949 & 11.24 & 1.13 \\
2453810.8555 & -26.81 & 1.14 \\
\hline
\end{tabular}


Table 4. Individual RAdial Velocities for T-LyR1-01662

\begin{tabular}{crr}
\hline \hline $\begin{array}{c}\text { HJD } \\
\text { (days })\end{array}$ & $\begin{array}{c}V_{\text {rad }} \\
\left(\mathrm{km} \mathrm{s}^{-1}\right)\end{array}$ & $\begin{array}{c}\sigma\left(V_{\mathrm{rad}}\right) \\
\left(\mathrm{km} \mathrm{s}^{-1}\right)\end{array}$ \\
\hline 2453661.7019 & -28.11 & 0.47 \\
2453663.6613 & 17.34 & 0.69 \\
2453666.6132 & -6.53 & 0.82 \\
2453684.5817 & 18.79 & 0.81 \\
2453688.5651 & 15.52 & 0.95 \\
2453691.5797 & -21.79 & 1.00 \\
2453694.5962 & -26.56 & 0.97 \\
2453837.9580 & -2.49 & 0.92 \\
2453838.9680 & -33.90 & 1.01 \\
2453840.9543 & 16.64 & 0.92 \\
2453842.9740 & -30.62 & 1.00 \\
2453866.9636 & 15.00 & 0.84 \\
2453871.9095 & -6.71 & 1.19 \\
2453987.6572 & -29.91 & 0.93 \\
2453992.6429 & -3.20 & 0.85 \\
\hline
\end{tabular}


Table 5. Individual RAdial Velocities For T-LyR0-08070

\begin{tabular}{crr}
\hline \hline $\begin{array}{c}\text { HJD } \\
\text { (days })\end{array}$ & $\begin{array}{c}V_{\text {rad }} \\
\left(\mathrm{km} \mathrm{s}^{-1}\right)\end{array}$ & $\begin{array}{c}\sigma\left(V_{\mathrm{rad}}\right) \\
\left(\mathrm{km} \mathrm{s}^{-1}\right)\end{array}$ \\
\hline 2453129.9811 & -47.72 & 3.95 \\
2453135.9131 & -45.10 & 4.41 \\
2453157.9641 & -39.31 & 2.90 \\
2453160.8934 & -26.61 & 2.05 \\
2453457.0047 & -47.04 & 3.93 \\
2453481.9792 & -23.26 & 3.01 \\
2453483.9629 & -61.90 & 4.02 \\
2453485.9666 & 4.96 & 2.71 \\
2453486.9020 & 13.84 & 2.42 \\
2453487.9680 & -6.14 & 2.98 \\
2453488.9413 & -48.73 & 2.76 \\
2453509.9377 & -55.56 & 3.90 \\
2453510.8796 & -1.92 & 2.58 \\
2453511.9144 & 13.61 & 2.18 \\
2453872.9397 & -30.64 & 6.23 \\
\hline
\end{tabular}


Table 6. Individual Radial Velocities For T-Cyg1-01385

\begin{tabular}{crr}
\hline \hline $\begin{array}{c}\text { HJD } \\
\text { (days })\end{array}$ & $\begin{array}{c}V_{\text {rad }} \\
\left(\mathrm{km} \mathrm{s}^{-1}\right)\end{array}$ & $\begin{array}{c}\sigma\left(V_{\text {rad }}\right) \\
\left(\mathrm{km} \mathrm{s}^{-1}\right)\end{array}$ \\
\hline 2453333.6202 & 8.44 & 1.17 \\
2453508.9739 & -38.15 & 1.19 \\
2453511.9801 & 24.75 & 0.91 \\
2453540.9615 & -38.52 & 1.72 \\
2453548.9596 & -24.14 & 1.33 \\
2453576.7477 & 18.30 & 1.13 \\
2453626.7511 & -41.05 & 1.33 \\
2453629.6988 & 22.52 & 1.00 \\
2453632.7413 & -36.62 & 1.64 \\
2453657.6540 & -2.19 & 1.12 \\
2453658.7019 & -30.46 & 1.10 \\
2453659.6389 & -40.07 & 1.45 \\
2453660.6781 & -20.03 & 1.40 \\
2453661.7327 & 11.68 & 1.57 \\
\hline
\end{tabular}

Table 7. Spectroscopic Orbital Solutions

\begin{tabular}{lllllll}
\hline \hline & & T-Aur0-13378 & T-Boo0-00080 & T-Lyr1-01662 & T-Lyr0-08070 & T-Cyg1-01385 \\
\hline N observations & & 14 & 18 & 13 & 16 & 15 \\
Time span & days & 802 & 732 & 380 & 863 & 656 \\
$P$ & days & $3.54182(14)$ & $2.539825(51)$ & $4.23339(22)$ & $1.184780(25)$ & $6.56012(29)$ \\
$\gamma$ & $\mathrm{km} \mathrm{s}^{-1}$ & $+11.47 \pm 0.44$ & $-12.97 \pm 0.30$ & $-7.42 \pm 0.19$ & $-25.49 \pm 0.98$ & $-6.81 \pm 0.17$ \\
$K$ & $\mathrm{~km} \mathrm{~s}^{-1}$ & $32.31 \pm 0.70$ & $32.81 \pm 0.38$ & $26.44 \pm 0.26$ & $42.87 \pm 1.50$ & $33.62 \pm 0.21$ \\
$e$ & & $0.040 \pm 0.022$ & $0.026 \pm 0.012$ & $0.037 \pm 0.010$ & $0.054 \pm 0.036$ & $0.023 \pm 0.007$ \\
$\omega$ & $\circ$ & $149 \pm 25$ & $78 \pm 25$ & $133 \pm 16$ & $346 \pm 74$ & $159 \pm 17$ \\
$O-C$ RMS & \multirow{2}{*}{$\mathrm{km} \mathrm{s}^{-1}$} & 1.47 & 1.06 & 0.73 & 3.38 & 0.54 \\
\hline
\end{tabular}


Table 8. KeplerCam Photometry

\begin{tabular}{|c|c|c|c|c|c|c|}
\hline & & T-Aur0-13378 & T-Boo0-00080 & T-Lyr1-01662 & T-Lyr0-08070 & T-Cyg1-01385 \\
\hline Date (UT) & & 2006 Dec 12 & 2006 Apr 17 & 2006 Jul 06 & 2006 Sep 18 & 2006 Jul 10 \\
\hline Band & & Sloan $i$ & Sloan $i$ & Sloan $i$ & Sloan $z$ & Sloan $z$ \\
\hline Exposure & sec & 30 & 30 & 30 & 45 & 30 \\
\hline Cadence & $\mathrm{im} / \mathrm{min}$ & 1.42 & 1.42 & 1.42 & 1.04 & 1.42 \\
\hline FWHM & $\operatorname{arcsec}$ & $1.4-1.9$ & $1.7-2.6$ & $1.4-1.9$ & $1.6-2.1$ & $1.4-2.7$ \\
\hline $\sec z$ & & $1.0-2.3$ & $1.7-1.0-1.2$ & $1.4-1.1-1.2$ & $1.0-1.7$ & $1.6-1.0-1.3$ \\
\hline
\end{tabular}

Table 9. KeplerCam Light Curves

\begin{tabular}{ccccc}
\hline \hline Object & Band & HJD & Relative Flux & $\sigma$ \\
\hline T-Aur0-13378 & i & 2454081.768872 & 0.9982 & 0.0016 \\
T-Aur0-13378 & i & 2454081.769381 & 0.9989 & 0.0016 \\
T-Aur0-13378 & i & 2454081.769890 & 0.9954 & 0.0016 \\
T-Aur0-13378 & i & 2454081.770400 & 0.9942 & 0.0016 \\
& & & & \\
T-Boo0-00080 & i & 2453842.652138 & 0.9998 & 0.0014 \\
T-Boo0-00080 & i & 2453842.652844 & 0.9986 & 0.0014 \\
T-Boo0-00080 & i & 2453842.653319 & 0.9974 & 0.0014 \\
T-Boo0-00080 & i & 2453842.653805 & 0.9996 & 0.0014 \\
& & & & \\
T-Lyr1-01662 & i & 2453922.652243 & 1.0001 & 0.0015 \\
T-Lyr1-01662 & i & 2453922.652729 & 0.9947 & 0.0015 \\
T-Lyr1-01662 & i & 2453922.653227 & 0.9967 & 0.0015 \\
T-Lyr1-01662 & i & 2453922.653690 & 1.0003 & 0.0015 \\
& & & & \\
T-Lyr0-08070 & z & 2453996.632634 & 0.9984 & 0.0015 \\
T-Lyr0-08070 & z & 2453996.633282 & 0.9982 & 0.0015 \\
T-Lyr0-08070 & z & 2453996.633954 & 0.9982 & 0.0015 \\
T-Lyr0-08070 & z & 2453996.634602 & 1.0014 & 0.0015 \\
& & & & \\
T-Cyg1-01385 & z & 2453926.673722 & 0.9976 & 0.0012 \\
T-Cyg1-01385 & z & 2453926.674324 & 1.0021 & 0.0012 \\
T-Cyg1-01385 & z & 2453926.675527 & 0.9966 & 0.0012 \\
T-Cyg1-01385 & z & 2453926.676129 & 1.0016 & 0.0012 \\
\hline
\end{tabular}

Note. - These are sample entries of the full light curves. The complete versions are given on-line. 
Table 10. Primary Star Spectroscopic Atmospheric Properties

\begin{tabular}{|c|c|c|c|c|c|c|}
\hline & & T-Aur0-13378 & T-Boo0-00080 & T-Lyr1-01662 & T-Lyr0-08070 & T-Cyg1-01385 \\
\hline \multicolumn{7}{|c|}{$[\mathrm{Fe} / \mathrm{H}]_{\text {adopted }}=-1.0$} \\
\hline$T_{\text {eff }}$ & $\mathrm{K}$ & $5860 \pm 100$ & $5510 \pm 30$ & $5810 \pm 30$ & $6000 \pm 150$ & $5520 \pm 30$ \\
\hline$V_{\text {rot }} \sin i_{\text {rot }}$ & $\mathrm{km} \mathrm{s}^{-1}$ & $25.3 \pm 0.7$ & $35.9 \pm 0.5$ & $13.0 \pm 0.4$ & $57.9 \pm 2.1$ & $11.9 \pm 0.6$ \\
\hline $\log g_{\mathrm{A}}$ & $\mathrm{cm} \mathrm{s}^{-2}$ & $3.79 \pm 0.02$ & $4.01 \pm 0.02^{\mathrm{a}}$ & $4.22 \pm 0.04$ & $4.13 \pm 0.01$ & $3.98 \pm 0.02^{\mathrm{a}}$ \\
\hline \multicolumn{7}{|c|}{$[\mathrm{Fe} / \mathrm{H}]_{\text {adopted }}=-0.5$} \\
\hline$T_{\text {eff }}$ & & $6200 \pm 80$ & $5850 \pm 30$ & $6200 \pm 30$ & $6250 \pm 140$ & $5580 \pm 30$ \\
\hline$V_{\text {rot }} \sin i_{\text {rot }}$ & $\mathrm{km} \mathrm{s}^{-1}$ & $25.7 \pm 0.7$ & $35.8 \pm 0.5$ & $13.6 \pm 0.4$ & $57.9 \pm 2.1$ & $12.6 \pm 0.6$ \\
\hline $\log g_{\mathrm{A}}$ & $\mathrm{cm} \mathrm{s}^{-2}$ & $3.83 \pm 0.02$ & $4.01 \pm 0.02$ & $4.25 \pm 0.03$ & $4.16 \pm 0.01$ & $3.98 \pm 0.02$ \\
\hline \multicolumn{7}{|c|}{$[\mathrm{Fe} / \mathrm{H}]_{\text {adopted }}=0.0$} \\
\hline$T_{\text {eff }}$ & & $6620 \pm 80$ & $6190 \pm 30$ & $6760 \pm 30$ & $6500 \pm 140$ & $6270 \pm 30$ \\
\hline$V_{\text {rot }} \sin i_{\text {rot }}$ & $\mathrm{km} \mathrm{s}^{-1}$ & $25.5 \pm 0.7$ & $35.8 \pm 0.5$ & $13.8 \pm 0.4$ & $57.8 \pm 2.1$ & $13.0 \pm 0.6$ \\
\hline $\log g_{\mathrm{A}}$ & $\mathrm{cm} \mathrm{s}^{-2}$ & $3.88 \pm 0.02$ & $4.06 \pm 0.02$ & $4.30 \pm 0.02$ & $4.21 \pm 0.01$ & $4.03 \pm 0.02$ \\
\hline \multicolumn{7}{|c|}{$[\mathrm{Fe} / \mathrm{H}]_{\text {adopted }}=+0.5$} \\
\hline$T_{\text {eff }}$ & $\mathrm{K}$ & $6990 \pm 80$ & $6610 \pm 30$ & $7210 \pm 40$ & $6710 \pm 140$ & $6710 \pm 30$ \\
\hline$V_{\text {rot }} \sin i_{\text {rot }}$ & $\mathrm{km} \mathrm{s}^{-1}$ & $25.5 \pm 0.7$ & $35.9 \pm 0.5$ & $14.4 \pm 0.4$ & $58.3 \pm 2.1$ & $13.4 \pm 0.6$ \\
\hline $\log g_{\mathrm{A}}$ & $\mathrm{cm} \mathrm{s}^{-2}$ & $3.91 \pm 0.02$ & $4.10 \pm 0.02$ & $4.30 \pm 0.02^{\mathrm{a}}$ & $4.23 \pm 0.01$ & $4.07 \pm 0.01$ \\
\hline
\end{tabular}

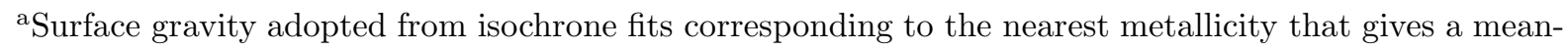
ingful result 
Table 11. Summary of Parameter of Transit Light Curve Analysis

\begin{tabular}{lllllll}
\hline \hline & & T-Aur0-13378 & T-Boo0-00080 & T-Lyr1-01662 & T-Lyr0-08070 & T-Cyg1-01385 \\
\hline Epoch & HJD & $2454081.8678(2)$ & $2453842.8212(2)$ & $2453922.7207(2)$ & $2453996.7368(2)$ & $2453926.8104(2)$ \\
Duration & min & $156.9 \pm 0.4$ & $194.1 \pm 0.2$ & $201.4 \pm 0.3$ & $176.6 \pm 0.2$ & $190.2 \pm 0.2$ \\
$u_{1}$ & & 0.1509 & 0.1917 & 0.1628 & 0.2467 & 0.1431 \\
$u_{2}$ & & 0.3834 & 0.3677 & 0.3776 & 0.3821 & 0.3594 \\
$a / R_{\mathrm{A}}$ & & $5.10 \pm 0.08$ & $5.21 \pm 0.03$ & $9.56 \pm 0.08$ & $3.69 \pm 0.01$ & $9.76_{-0.08}^{+0.05}$ \\
$R_{\mathrm{B}} / R_{\mathrm{A}}$ & & $0.1551(7)$ & $0.1775(8)$ & $0.2085(8)$ & $0.1952(4)$ & $0.2205(3)$ \\
$b$ & $0.63 \pm 0.02$ & $0.815 \pm 0.004$ & $0.716 \pm 0.007$ & $0.05_{-0.03}^{+0.05}$ & $0.11 \pm 0.06$ \\
$O-C$ RMS & $\%$ & 0.21 & 0.10 & 0.16 & 0.18 & 0.22 \\
\hline & & & & & \\
$\rho_{\mathrm{A}}{ }^{a}$ & $\mathrm{gr} \mathrm{cm}^{-3}$ & $0.163 \pm 0.008$ & $0.344 \pm 0.006$ & $0.75 \pm 0.02$ & $0.542 \pm 0.008$ & $0.297 \pm 0.007$ \\
$\log g_{\mathrm{B}}{ }^{a}$ & $\mathrm{~cm} \mathrm{~s}^{-2}$ & $4.86 \pm 0.02$ & $4.91 \pm 0.01$ & $4.98 \pm 0.01$ & $4.97 \pm 0.02$ & $4.86 \pm 0.01$ \\
\hline
\end{tabular}

aThese quantities are essentially model-independent and rely only on spectroscopic and photometric observables. The dependence of $\rho_{\mathrm{A}}$ on $\rho_{\mathrm{B}}$ is typically very weak as the secondary star is usually small compared to the primary (See Eq. [5]. 
Table 12. Results Derived from Stellar Isochrones

\begin{tabular}{|c|c|c|c|c|c|c|}
\hline & & T-Aur0-13378 & T-Boo0-00080 a & T-Lyr1-01662 a & T-Lyr0-08070 & T-Cyg1-01385 a \\
\hline \multicolumn{7}{|c|}{$[\mathrm{Fe} / \mathrm{H}]_{\text {adopted }}=-1.0 \pm 0.2$} \\
\hline Age & Gyr & $9.0 \pm 3.0$ & - & $12.9 \pm 1.5$ & $12.6 \pm 1.8$ & - \\
\hline$M_{\mathrm{A}}$ & $M_{\odot}$ & $0.92 \pm 0.11$ & - & $0.81 \pm 0.20$ & $0.82 \pm 0.06$ & - \\
\hline$R_{\mathrm{A}}$ & $R_{\odot}$ & $2.03 \pm 0.08$ & - & $1.16 \pm 0.08$ & $1.30 \pm 0.03$ & - \\
\hline$M_{\mathrm{B}}$ & $M_{\odot}$ & $0.26 \pm 0.02$ & - & $0.20 \pm 0.03$ & $0.22 \pm 0.02$ & - \\
\hline$R_{\mathrm{B}}$ & $R_{\odot}$ & $0.31 \pm 0.02$ & - & $0.24 \pm 0.02$ & $0.25 \pm 0.01$ & - \\
\hline \multicolumn{7}{|c|}{$[\mathrm{Fe} / \mathrm{H}]_{\text {adopted }}=-0.5 \pm 0.2$} \\
\hline Age & Gyr & $4.5 \pm 1.3$ & $10.4 \pm 3.7$ & $7.7 \pm 4.8$ & $6.9 \pm 1.7$ & $9.5 \pm 2.6$ \\
\hline$M_{\mathrm{A}}$ & $M_{\odot}$ & $1.18 \pm 0.14$ & $0.93 \pm 0.15$ & $0.96 \pm 0.18$ & $1.00 \pm 0.08$ & $0.95 \pm 0.10$ \\
\hline$R_{\mathrm{A}}$ & $R_{\odot}$ & $2.18 \pm 0.08$ & $1.58 \pm 0.07$ & $1.22 \pm 0.07$ & $1.38 \pm 0.03$ & $1.66 \pm 0.05$ \\
\hline$M_{\mathrm{B}}$ & $M_{\odot}$ & $0.30 \pm 0.02$ & $0.24 \pm 0.02$ & $0.23 \pm 0.03$ & $0.25 \pm 0.02$ & $0.35 \pm 0.02$ \\
\hline$R_{\mathrm{B}}$ & $R_{\odot}$ & $0.34 \pm 0.02$ & $0.28 \pm 0.02$ & $0.25 \pm 0.02$ & $0.27 \pm 0.01$ & $0.37 \pm 0.01$ \\
\hline \multicolumn{7}{|c|}{$[\mathrm{Fe} / \mathrm{H}]_{\text {adopted }}=0.0 \pm 0.2$} \\
\hline Age & Gyr & $2.0 \pm 1.5$ & $4.1 \pm 1.7$ & $0.8 \pm 0.7$ & $2.2 \pm 0.7$ & $3.7 \pm 1.5$ \\
\hline$M_{\mathrm{A}}$ & $M_{\odot}$ & $1.60 \pm 0.13$ & $1.26 \pm 0.17$ & $1.35 \pm 0.08$ & $1.33 \pm 0.07$ & $1.31 \pm 0.16$ \\
\hline$R_{\mathrm{A}}$ & $R_{\odot}$ & $2.40 \pm 0.10$ & $1.74 \pm 0.07$ & $1.35 \pm 0.04$ & $1.50 \pm 0.03$ & $1.82 \pm 0.06$ \\
\hline$M_{\mathrm{B}}$ & $M_{\odot}$ & $0.37 \pm 0.03$ & $0.28 \pm 0.02$ & $0.28 \pm 0.02$ & $0.29 \pm 0.02$ & $0.43 \pm 0.03$ \\
\hline$R_{\mathrm{B}}$ & $R_{\odot}$ & $0.37 \pm 0.02$ & $0.31 \pm 0.02$ & $0.28 \pm 0.01$ & $0.29 \pm 0.01$ & $0.40 \pm 0.02$ \\
\hline \multicolumn{7}{|c|}{$[\mathrm{Fe} / \mathrm{H}]$ adopted $=+0.5 \pm 0.2$} \\
\hline Age & Gyr & $0.9 \pm 0.2$ & $1.2 \pm 0.5$ & - & $0.5 \pm 0.1$ & $1.0 \pm 0.4$ \\
\hline$M_{\mathrm{A}}$ & $M_{\odot}$ & $1.91 \pm 0.07$ & $1.58 \pm 0.06$ & - & $1.52 \pm 0.02$ & $1.64 \pm 0.06$ \\
\hline$R_{\mathrm{A}}$ & $R_{\odot}$ & $2.54 \pm 0.05$ & $1.86 \pm 0.02$ & - & $1.57 \pm 0.01$ & $1.95 \pm 0.03$ \\
\hline$M_{\mathrm{B}}$ & $M_{\odot}$ & $0.41 \pm 0.01$ & $0.33 \pm 0.01$ & - & $0.32 \pm 0.01$ & $0.49 \pm 0.01$ \\
\hline$R_{\mathrm{B}}$ & $R_{\odot}$ & $0.39 \pm 0.01$ & $0.33 \pm 0.01$ & - & $0.31 \pm 0.01$ & $0.43 \pm 0.01$ \\
\hline
\end{tabular}

${ }^{a}$ No meaningful solution found for T-Boo0-00080, T-Lyr1-01662 and T-Cyg1-01385 when adopting a very low or high metallicity for the primary star. 
Table 13. Results Derived from Orbit-Rotation Synchronization

\begin{tabular}{|c|c|c|c|c|c|c|}
\hline & & T-Aur0-13378 a & T-Boo0-00080 & T-Lyr1-01662 & T-Lyr0-08070 & T-Cyg1-01385 \\
\hline$[\mathrm{Fe} / \mathrm{H}]$ adopted $\mathrm{b}$ & & - & +0.5 & -0.5 & -0.5 & -0.5 \\
\hline$M_{\mathrm{A}(\mathrm{sync})}$ & $M_{\odot}$ & - & $1.49 \pm 0.07$ & $0.77 \pm 0.08$ & $0.95 \pm 0.11$ & $0.91 \pm 0.15$ \\
\hline$R_{\mathrm{A}(\text { sync })}$ & $R_{\odot}$ & - & $1.83 \pm 0.03$ & $1.14 \pm 0.03$ & $1.36 \pm 0.05$ & $1.63 \pm 0.08$ \\
\hline $\log g_{\mathrm{A}(\text { sync })}$ & $\mathrm{cm} \mathrm{s}^{-2}$ & - & $4.09 \pm 0.01$ & $4.21 \pm 0.02$ & $4.15 \pm 0.02$ & $3.97 \pm 0.03$ \\
\hline$M_{\mathrm{B}(\mathrm{sync})}$ & $M_{\odot}$ & - & $0.315 \pm 0.010$ & $0.198 \pm 0.012$ & $0.240 \pm 0.019$ & $0.345 \pm 0.034$ \\
\hline$R_{\mathrm{B}(\mathrm{sync})}$ & $R_{\odot}$ & - & $0.325 \pm 0.005$ & $0.238 \pm 0.007$ & $0.265 \pm 0.010$ & $0.360 \pm 0.017$ \\
\hline
\end{tabular}

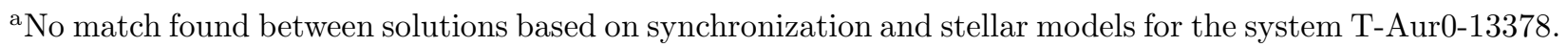

${ }^{\mathrm{b}}$ Metallicity for the best match between the solutions based on synchronization and stellar models. 


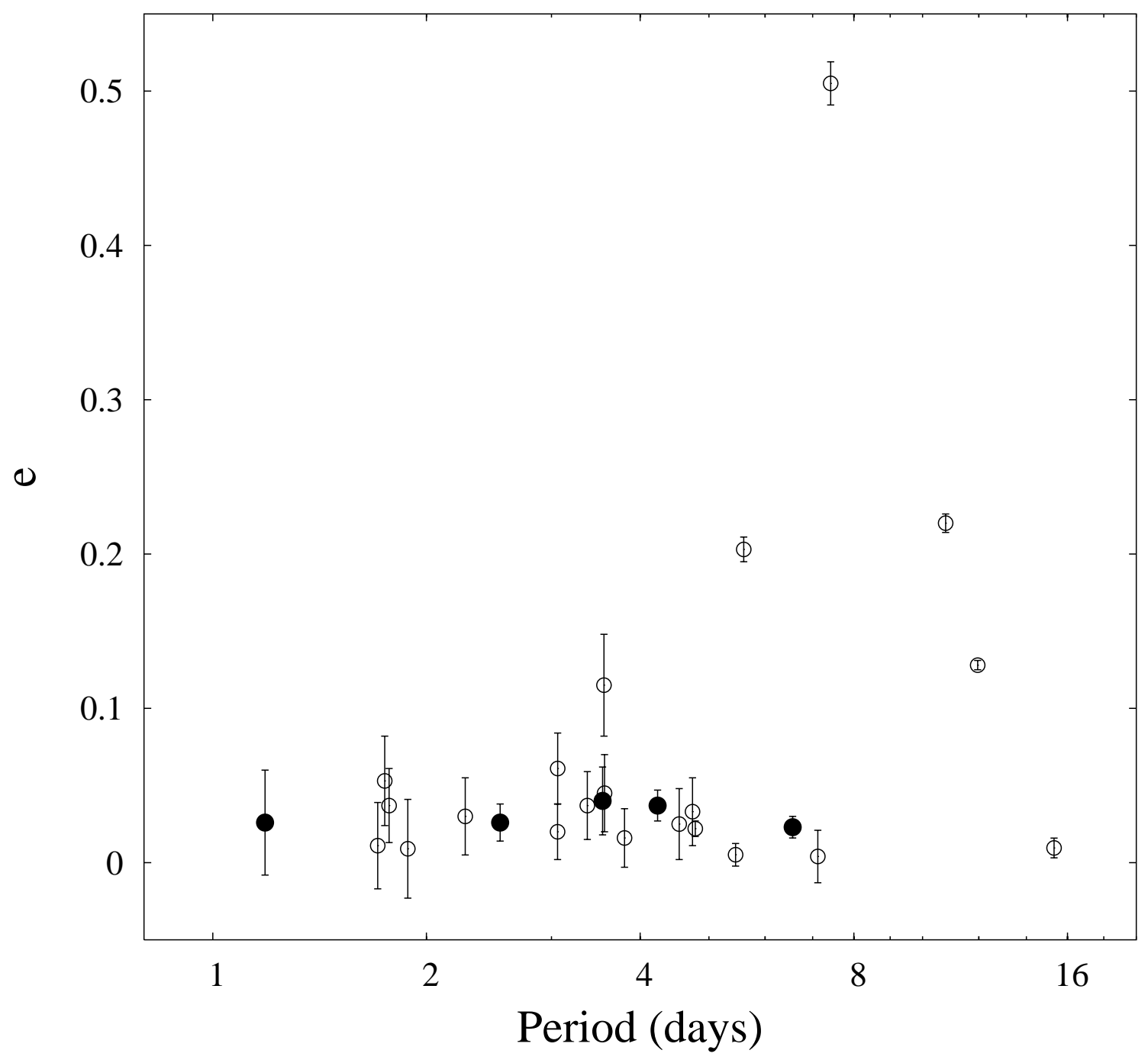

Fig. 1.- Eccentricity vs. orbital period diagram for 26 single-lined eclipsing binary stars discovered by TrES. The five systems presented in this work are shown as filled circles. 


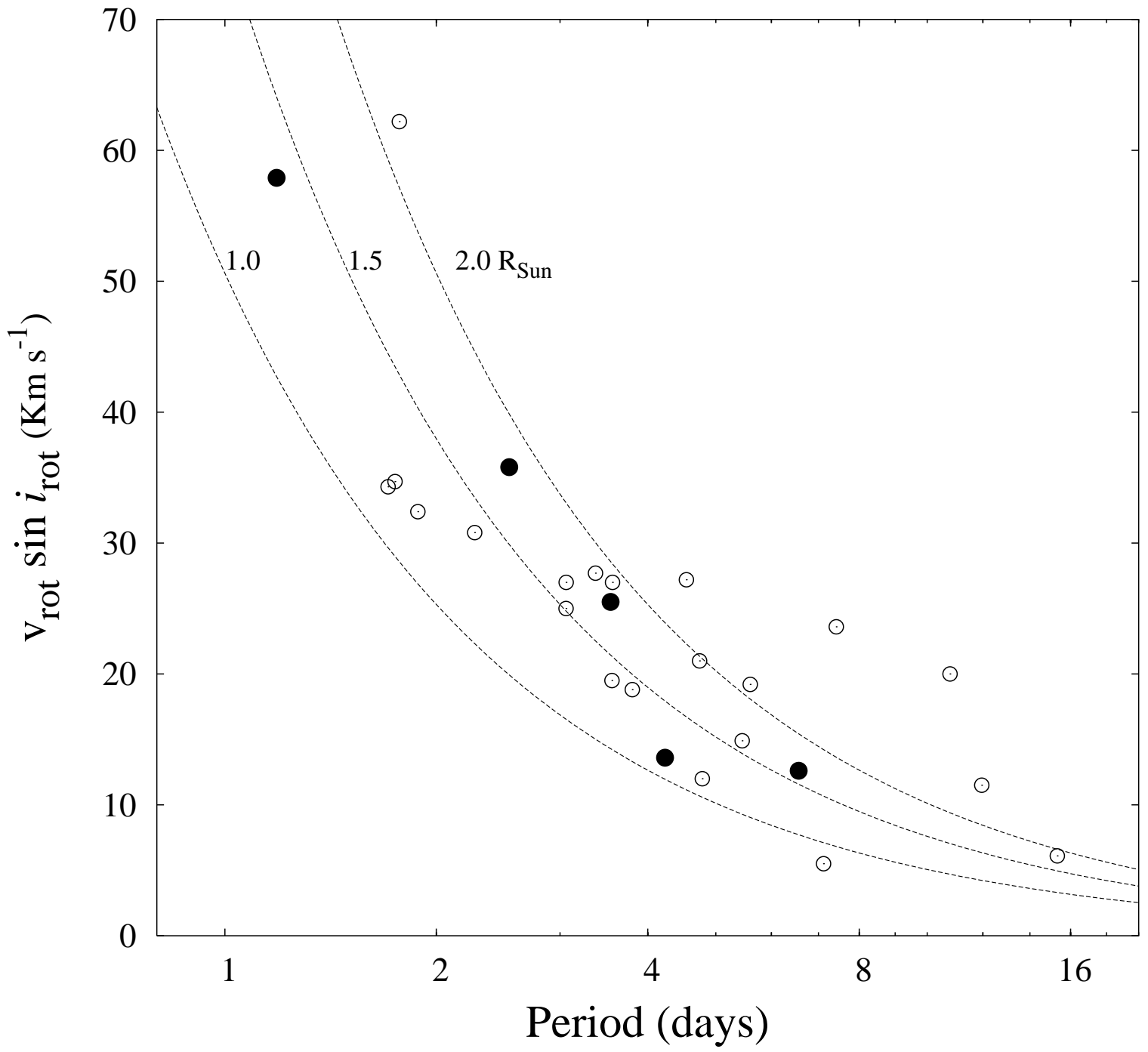

Fig. 2.- Rotational velocity vs. orbital period for 26 SEB's discovered by TrES. Curves of constant radius in solar units are shown, computed under the assumption of orbit-rotation synchronization. The five systems studied in this work are shown as filled circles. 


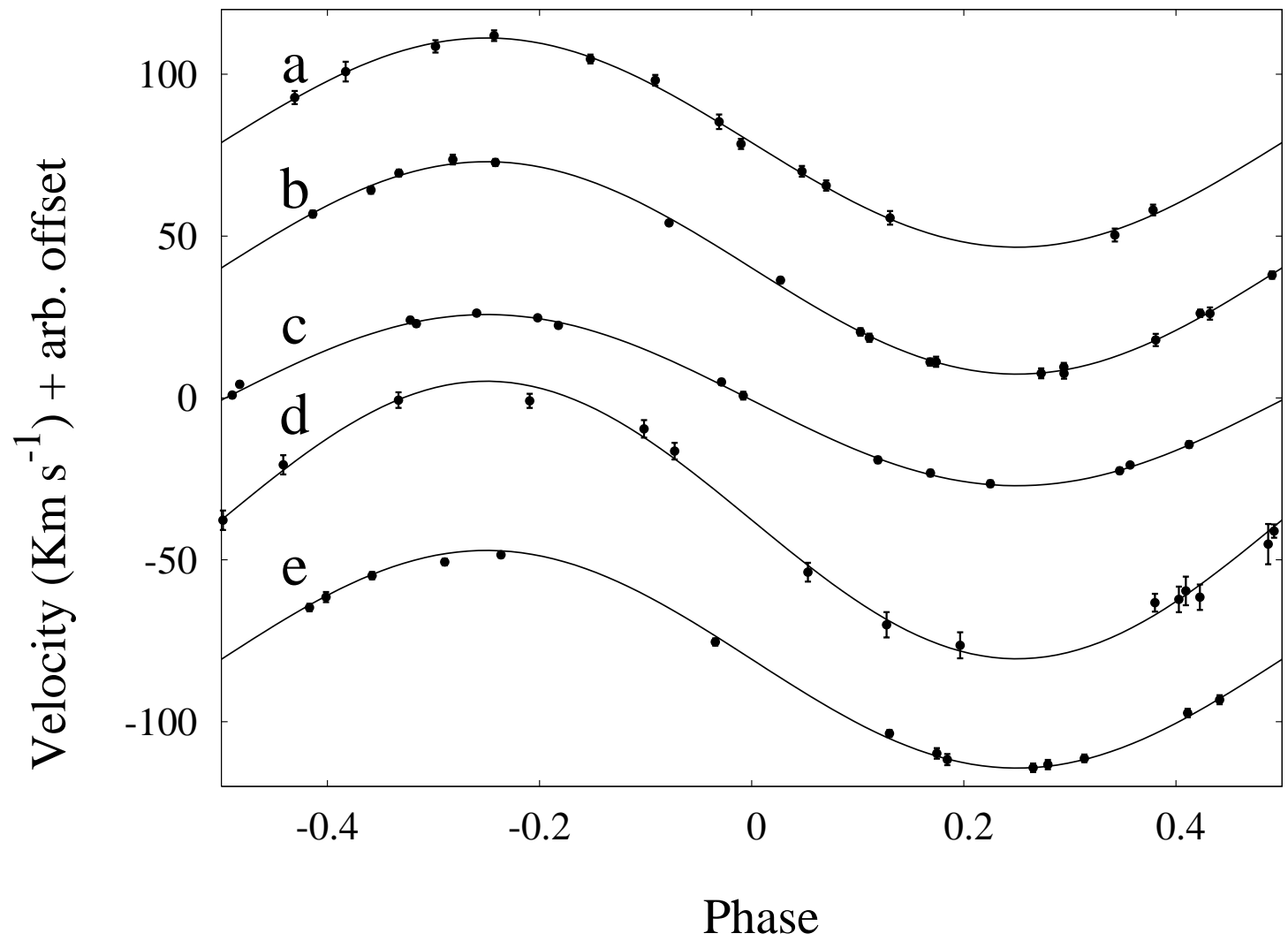

Fig. 3.- Period-phased radial velocities for T-Aur0-13378 (a), T-Boo0-00080 (b) T-Lyr101662 (c), T-Lyr0-08070 (d) and T-Cyg1-01385 (e). Continuous lines show the best orbital fit for each data set. 


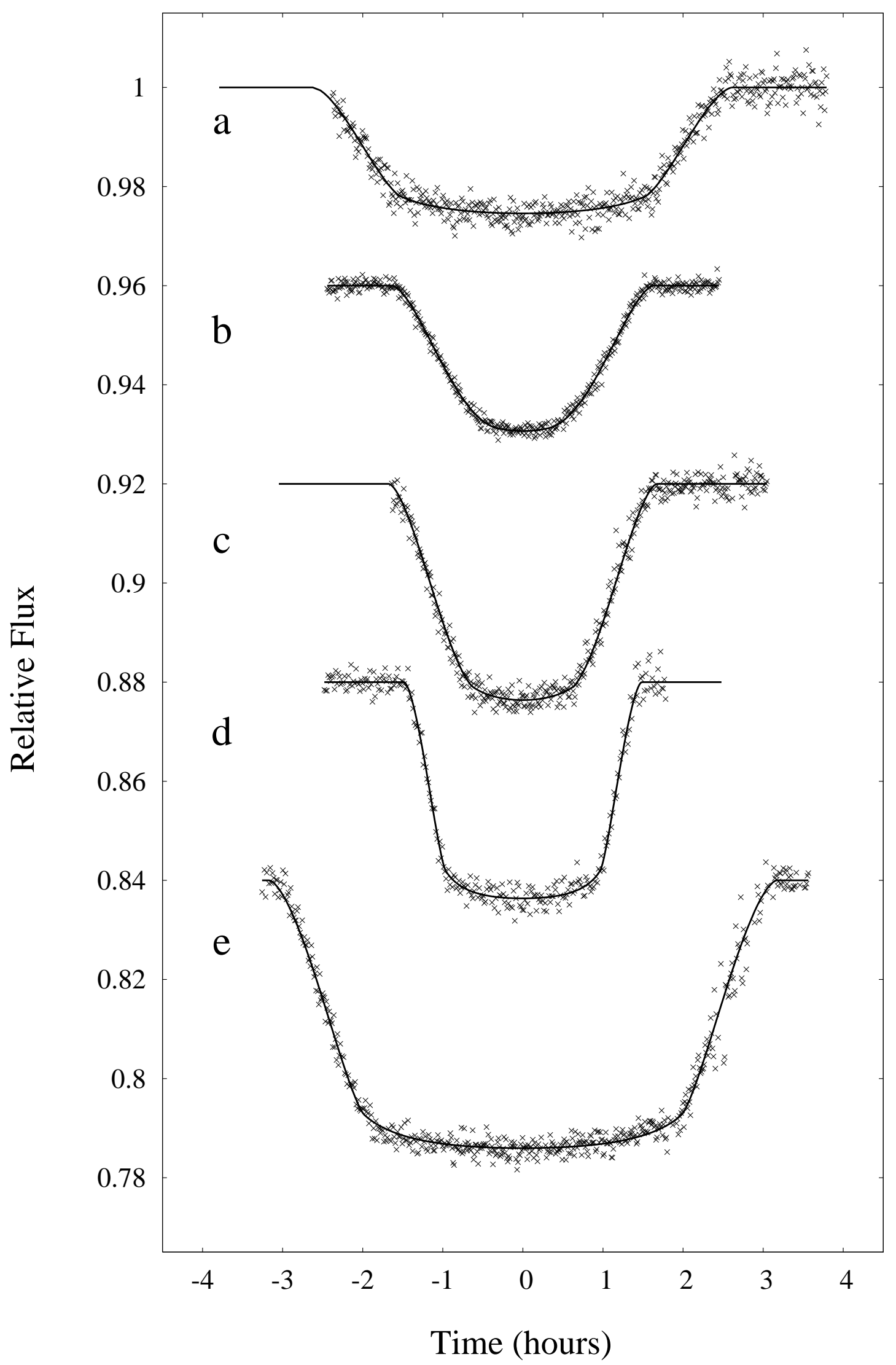

Fig. 4.- KeplerCam primary transit light curves for T-Aur0-13378 (a), T-Boo0-00080 (b) 

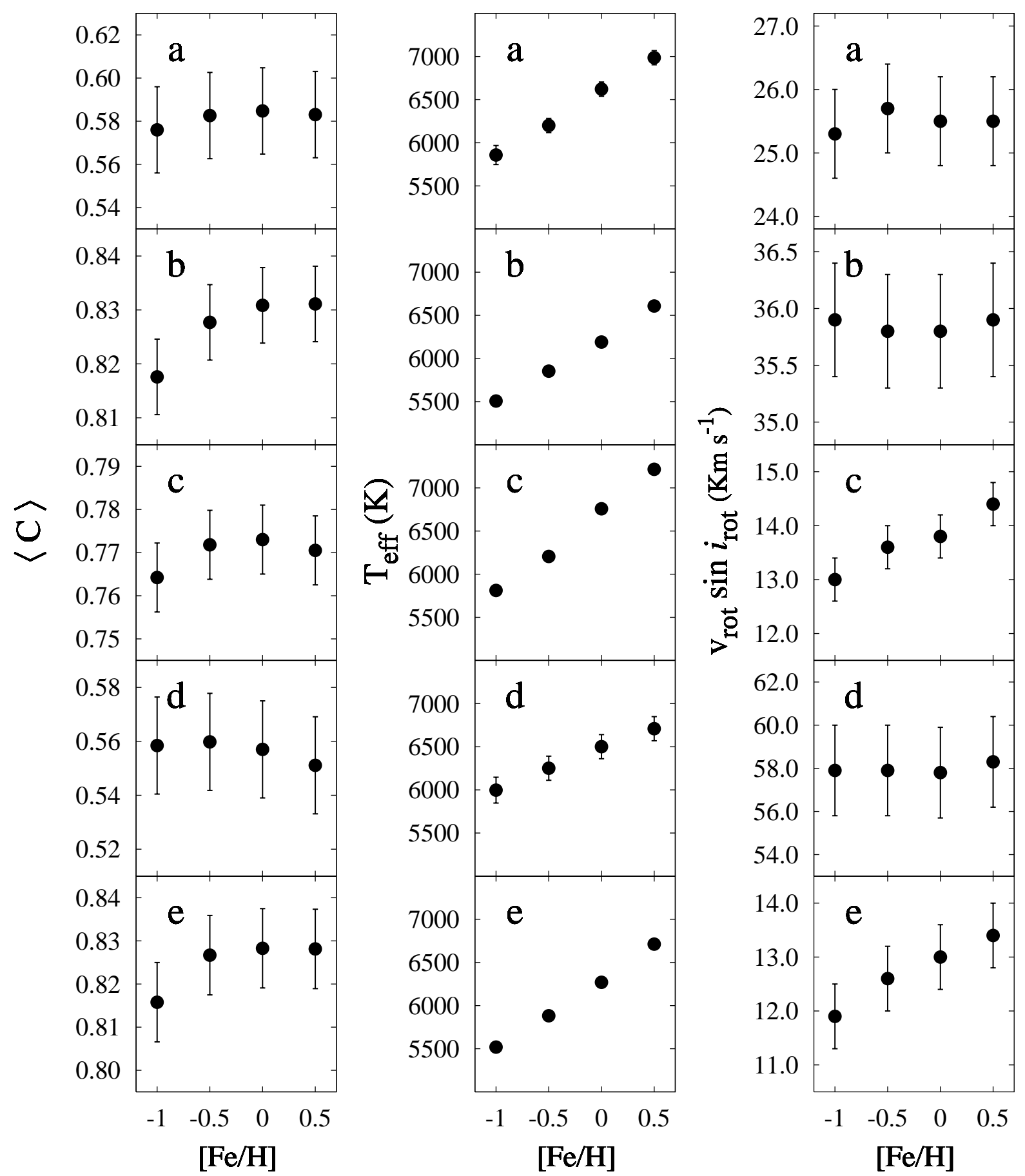

Fig. 5.- Dependence of the cross-correlation index, temperature and projected rotational velocity on the adopted metallicity for T-Aur0-13378 (a), T-Boo0-00080 (b) T-Lyr1-01662 (c), T-Lyr0-08070 (d) and T-Cyg1-01385 (e). 

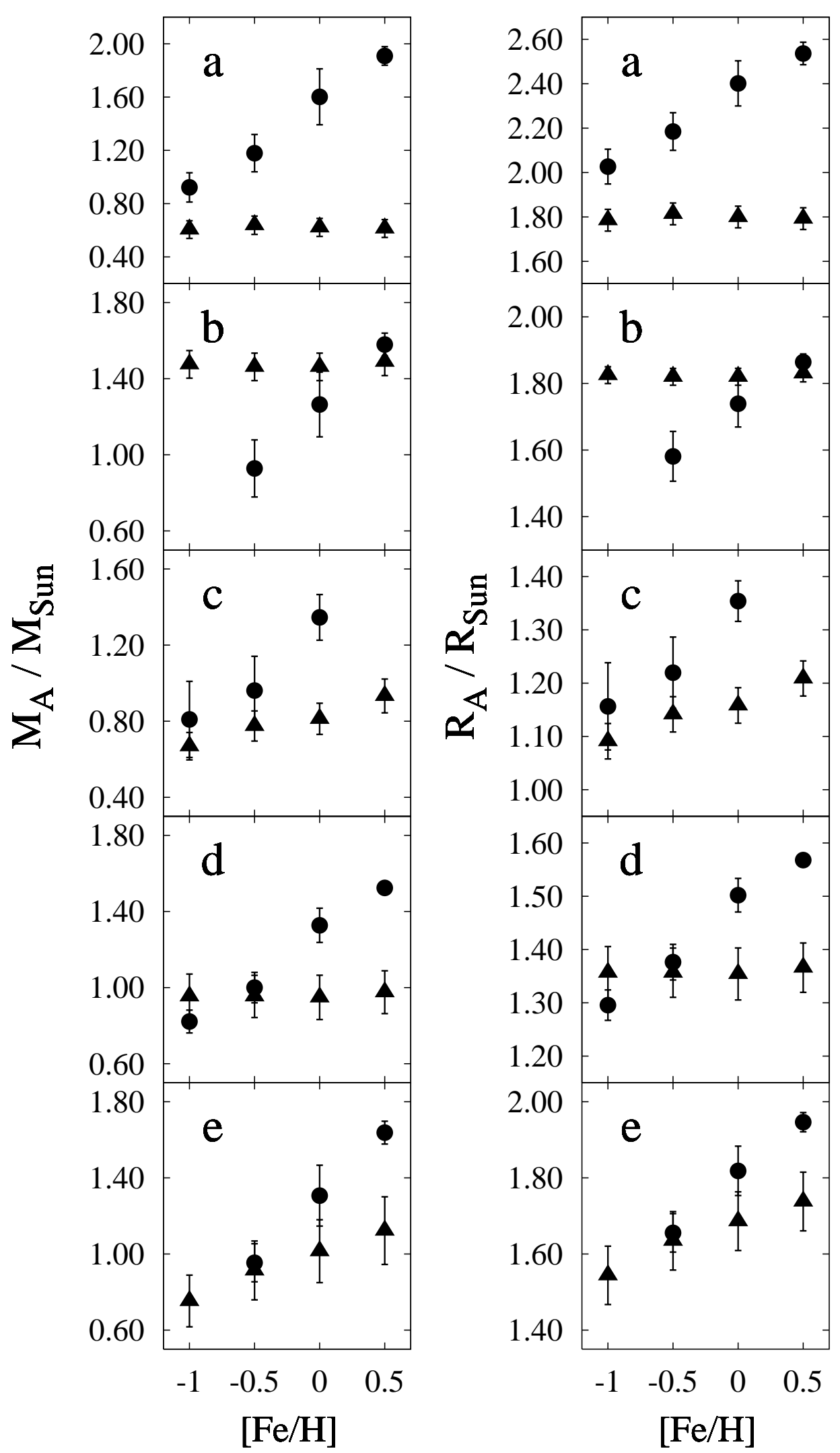

Fig. 6. - Dependence of the mass and radius of the primary star on the adopted metallicity, for T-Aur0-13378 (a), T-Boo0-00080 (b) T-Lyr1-01662 (c), T-Lyr0-08070 (d) and T-Cyg101385 (e). Results derived from stellar isochrones are shown as filled circles. while the results 


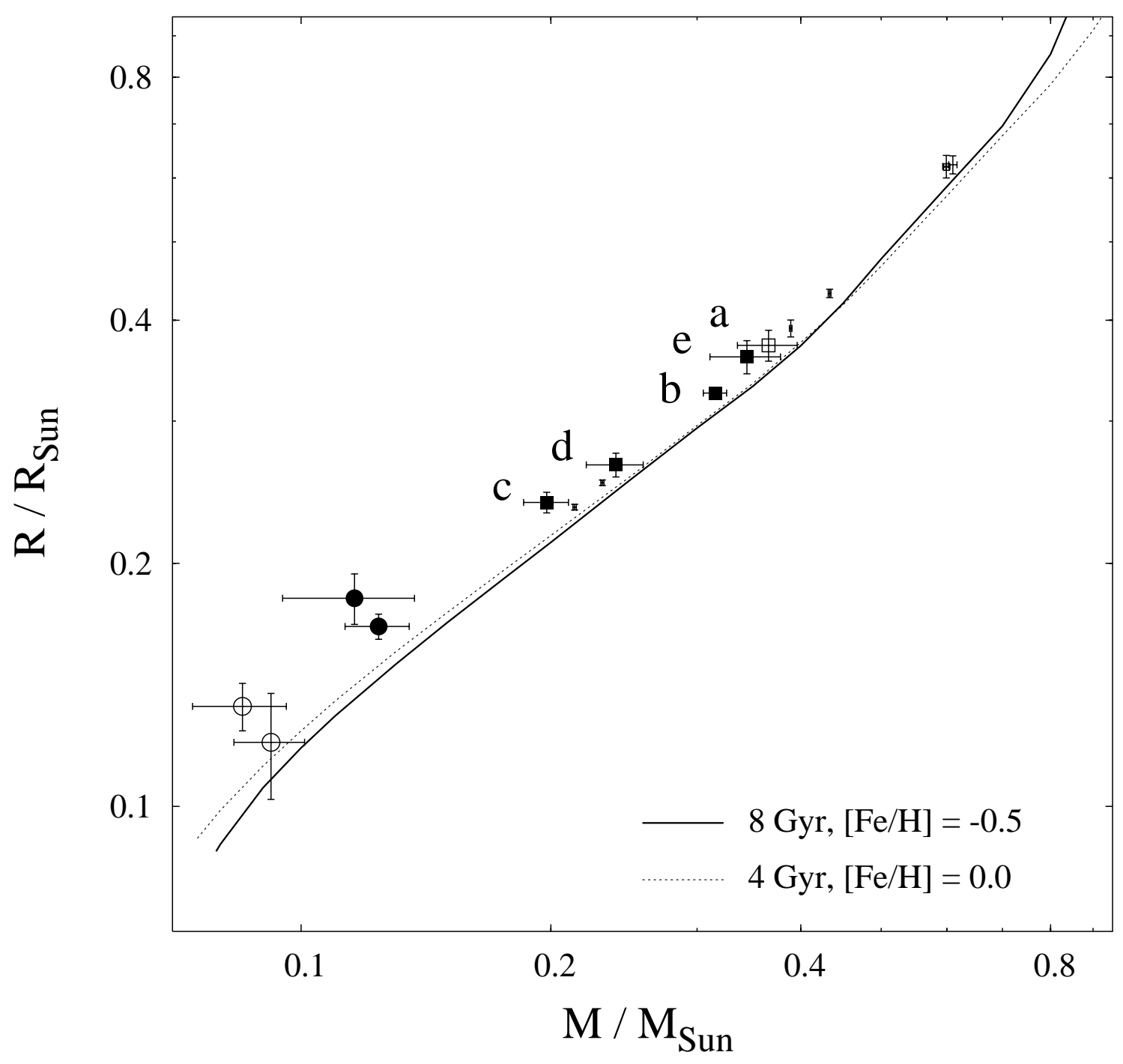

Fig. 7.- Mass-radius diagram for the M dwarfs T-Aur0-13378 (a), T-Boo0-00080-B (b), TLyr1-01662-B (c), T-Lyr0-08070-B (d) and T-Cyg1-01385-B (e), shown as squares. Four additional M dwarfs in SEBs studied by other authors are shown as circles (Pont et al. 2005a, b, 2006; Beatty et al. 2007). Filled symbols correspond to results derived from the assumption of orbit-rotation synchronization, and open symbols correspond to results derived from stellar models. Eight M dwarfs in double-lined eclipsing binaries (four systems total) are shown as dots (Morales et al. 2008; Torres \& Ribas 2002; Ribas 2003; López-Morales \& Ribas 2005) together with low-mass stellar isochrones (Baraffe et al. 1998). 